\title{
Les parois chauffées de la grotte Chauvet-Pont d'Arc (Ardèche, France) : caractérisation et chronologie
}

Heated walls of the cave Chauvet-Pont d'Arc (Ardèche, France) : characterization and chronology

Catherine Ferrier, Évelyne Debard, Bertrand Kervazo, Aurélie Brodard, Pierre Guibert, Dominique Baffier, Valérie Feruglio, Bernard Gély, JeanMichel Geneste et Frédéric Maksud

\section{OpenEdition Journals}

Édition électronique

URL : http://journals.openedition.org/paleo/2730

DOI : $10.4000 /$ paleo. 2730

ISSN : 2101-0420

Éditeur

SAMRA

Édition imprimée

Date de publication : 28 décembre 2014

Pagination : 59-78

ISSN : 1145-3370

Référence électronique

Catherine Ferrier, Évelyne Debard, Bertrand Kervazo, Aurélie Brodard, Pierre Guibert, Dominique Baffier, Valérie Feruglio, Bernard Gély, Jean-Michel Geneste et Frédéric Maksud, «Les parois chauffées de la grotte Chauvet-Pont d'Arc (Ardèche, France) : caractérisation et chronologie », PALEO [En ligne], 25 2014, mis en ligne le 31 juillet 2015, consulté le 07 juillet 2020. URL : http://journals.openedition.org/ paleo/2730 ; DOI : https://doi.org/10.4000/paleo.2730

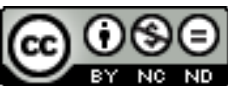

PALEO est mis à disposition selon les termes de la licence Creative Commons Attribution - Pas d'Utilisation Commerciale - Pas de Modification 4.0 International. 


\title{
Les parois chauffées de la grotte Chauvet-Pont d'Arc (Ardèche, France) : caractérisation et chronologie
}

\author{
Catherine FERRIER(1), Évelyne DEBARD ${ }^{(2)}$, Bertrand KERVAZO(3,1), \\ Aurélie BRODARD ${ }^{(4)}$, Pierre GUIBERT ${ }^{(4)}$, Dominique BAFFIER ${ }^{(5)}$, \\ Valérie FERUGLIO(6), Bernard GÉLY(7), Jean-Michel GENESTE ${ }^{(3,1)}$, \\ Frédéric MAKSUD(8)
}

\begin{abstract}
Résumé : Les recherches sur la taphonomie des parois ornées de la grotte Chauvet-Pont d'Arc ont conduit à l'identification et à l'étude détaillée de traces résultant d'un processus de chauffe : teintes rose et grise de la roche, écaillage et dépôts dus à la fumée. Ce faciès thermique a été observé aussi bien dans les premières salles, comportant majoritairement des peintures rouges, que dans les salles profondes où se trouvent la plupart des représentations réalisées au charbon de bois. Une étude paléothermométrique par thermoluminescence a été mise en œuvre sur des fragments de calcaire rubéfié collectés au sol ou sur les parois. Elle montre que la roche a été chauffée dans le passé à des températures comprises entre 300 et $375^{\circ} \mathrm{C}$ selon l'échantillon. Les données chronologiques, tant absolues (datation C14 des charbons) que relatives (relation avec les représentations pariétales) indiquent que les feux se rapportent principalement à l'Aurignacien. La fonction de ces feux reste inconnue : éclairage, ravivage de torche, production de colorant, ou encore de fumée ou de chaleur sans nécessité directe autre que symbolique, modification de l'état de surface de la paroi, protection vis-à-vis des ours qui fréquentaient la grotte en même temps que les hommes...

Mots-clés : Chauvet-Pont d'Arc, taphonomie, thermo-altération, thermoluminescence, paléothermomètre, chronologie, radiocarbone.

Abstract: Heated walls of the cave Chauvet-Pont d'Arc (Ardèche, France): characterization and chronology. Researches on the taphonomy of the rock art walls of the Chauvet-Pont d'Arc cave led to the identification and the detailed study of marks resulting from a heating process: pink and grey color of the rock, flakes due to the heat, and soot deposits. This thermal facies was observed in the first rooms, where the main red paintings are located, as well as in the deep rooms where most of the charcoal paintings were found. A paleothermometric study was undertaken by a thermoluminescence analysis of reddened limestone chips sampled on the archaeological floor or extracted from the walls. As a result, this study showed that the surface of the rock was heated in the past at temperatures ranging from 300 to $375^{\circ} \mathrm{C}$ according to the sample. The chronological data (C14 datings of the charcoals and relative chronology with the rock art) link the fires mostly to the Aurignacian. The function of the fires remains unknown: lighting, getting the torch going again, colourant production, smoke, heat production, without other reason than symbolic, modification of the wall state, protection from the bears who were present in the cave at the same period...
\end{abstract}

Key-words: Chauvet-Pont d'Arc cave, taphonomy, thermal alteration, thermoluminescence, paleothermometry, chronology, radiocarbon dating.

(1) Université de Bordeaux, UMR 5199, PACEA, FR-33400 Talence cedex - c.ferrier@pacea.u-bordeaux1.fr

(2) Université Lyon 1, UMR 5276, Laboratoire de géologie de Lyon, FR-69622 Villeurbanne cedex - evelyne.debard@univ-lyon1.fr

$(3,1)$ Ministère de la Culture et de la Communication, Sous-direction de l'Archéologie, Centre National de Préhistoire, FR-24000

Périgueux - PACEA, UMR 5199 F-33400 Talence cedex - bertrand.kervazo@culture.gouv.fr ; jean-michel.geneste@culture.gouv.fr

(4) IRAMAT-CRP2A, UMR 5060, FR-33607 Pessac - pierre.guibert@u-bordeaux-montaigne.fr ; aurelie.brodard@gmail.fr

(5) CREPS, ArScAn, UMR 7041, FR-07150 Vallon-Pont d'Arc-dominique.baffier@culture.gouv.fr

(6) ArScAn, Ethnologie préhistorique, UMR 7041 - feruglio@free.fr

(7) SRA Rhône-Alpes, FR-69283 Lyon cedex 01 - bernard.gely@culture.gouv.fr

(8) SRA Midi-Pyrénées, FR-31200 Toulouse - frederic.maksud@culture.gouv.fr 


\section{Introduction}

En France, d'assez nombreuses grottes ornées comportent des accumulations de charbons pour lesquelles différentes fonctions ont été évoquées. Les mentions d'aires de combustion interprétées comme foyers domestiques sont rares et cantonnées aux grottes pyrénéennes telles que le Tuc d'Audoubert (Begouën et al. 2009), la grotte de Fontanet (Wahl 1973 ; Clottes 1977) et la grotte de Labastide (Omnès 1982 ; Delibrias et al. 1980). Dans ces deux cas, il s'agit d'occupations magdaléniennes. Les foyers datés du Solutréen et/ou du Gravettien, reconnus à Arcy-sur-Cure et à Cosquer, ont été interprétés comme des structures destinées à l'éclairage, au balisage du cheminement, à l'allumage des torches ou encore à la production de colorant (Baffier et al. 1998 ; Clottes et al. 2005). Les charbons dispersés au sol sont plus fréquemment cités et concernent notamment des grottes du Bassin parisien (Arcy-sur-Cure), des Pyrénées (Tuc d'Audoubert) et du Sud-Est (Aldène, Tête du Lion, DeuxOuvertures, Baume Latrone et Cosquer). Ils se rapportent à des périodes couvrant l'Aurignacien, le Gravettien, le Solutréen et le Magdalénien (Baffier et al. 1998 ; Begouën et al. 2009 ; Ambert et al. 2005 ; Combier 1984, 1989 ; Azéma et al. 2012 ; Gély 2000 ; Clottes et al. 2005 ; Philippe et al. 2009). II s'agit principalement de foyers remaniés, de vidanges de foyer ou d'escarbilles tombées de torches.

Des traces de chauffe (rubéfaction) sont parfois indiquées pour le sol - sédiment ou roche - (Baffier et al. 1998 ; Clottes et al. 2005 ; Begouën et al. 2009 par ex.) mais jamais pour les parois ou les plafonds situés à proximité des foyers.

Dans la grotte Chauvet, la présence de foyers a été reconnue dès la découverte, puis confirmée lors de l'expertise réalisée par J. Clottes (Chauvet et al. 1995). L'association de certaines aires de combustion avec des traces de rubéfaction et de desquamation rapportées à de violents chocs thermiques (Galerie des Mégacéros et des Croisillons) a ensuite été mentionnée par J.-M. Geneste (2001, 2005). Puis, les recherches entreprises à partir de 2008 sur la taphonomie des parois ornées ont permis :

- de reconnaître des traces identiques dans le Secteur d'Entrée, dans le Diverticule des Ours et à plusieurs endroits de la Galerie des Mégacéros (Debard et al. 2012 ; Ferrier et al. 2012) ;

- de déterminer les paléotempératures atteintes, à partir de l'étude par thermoluminescence d'échantillons prélevés dans ces secteurs (Brodard et al. 2014);

- de discuter de l'attribution chronologique des feux.

\section{Caractéristiques macroscopiques}

Dans la grotte Chauvet-Pont d'Arc, les secteurs présentant des marques de chauffe se caractérisent par (tabl. 1, fig. 1) :

- des colorations superficielles différentes de celle du calcaire. Ce sont surtout des teintes roses (rubéfaction) associées dans certains cas à des plages grises. La modification de la couleur peut affecter la roche sur des profondeurs estimées à plusieurs millimètres. Sur les plages concernées, elle ne s'exprime pas sur les coquilles des rudistes contenues dans le calcaire, montrant que le changement de teinte est lié à la présence des insolubles, notamment les oxydes et hydroxydes de fer ;

- un écaillage qui affecte la roche sur des surfaces variables, parfois supérieures au mètre carré. Les clastes qui en résultent sont soit des plaquettes minces, de $0,5 \mathrm{~cm}$ à $2 \mathrm{~cm}$ d'épaisseur et de longueur pluricentimétrique, soit des blocs dont l'épaisseur peut dépasser $10 \mathrm{~cm}$. Ces derniers se détachent à la faveur de fissures préexistantes dans le calcaire, de largeur millimétrique, souvent colmatées par des argiles brunes. Les cicatrices des écailles correspondent à des surfaces planes en général blanches, délimitées par des arêtes aiguës. Ces caractéristiques tranchent avec la forme et la couleur du reste de la paroi : microformes adoucies héritées des processus de dissolution lors des différentes phases de la karstogenèse, coloration blanche, orangée liée à la présence de revêtements argileux ou encore grise, due à la néogenèse de phosphates (Ferrier et al. 2012). Lorsque l'écaille a une faible épaisseur, de l'ordre de quelques millimètres, la cicatrice peut être localement colorée en rose, révélant la progression de la rubéfaction à l'intérieur de la roche;

- des liserés noirs majoritairement subhorizontaux, d'épaisseur infra-millimétrique, situés sur les irrégularités de la roche. Ils pourraient être interprétés comme des dépôts dus à la fumée.

L'ensemble de ces caractères définit un aspect superficiel du calcaire, lié à l'impact du feu, qualifié de faciès thermique. II renvoie à des observations réalisées de longue date sur les roches (calcaire ou non) et les sédiments archéologiques ayant subi une chauffe dans un foyer ou un four (Bodu et al. 2006 ; Foucher et al. 2000 ; Guilbert 2001 ; Mandeville 1973 ; Weisgerber et al. 2001).

\section{Répartition dans la grotte}

Ce faciès thermique a été observé à divers endroits de la grotte, aussi bien dans les premières salles, comportant majoritairement des peintures rouges faites avec des oxydes de fer, que dans les salles profondes où se trouvent la plupart des peintures noires réalisées avec des charbons de bois (fig. 2).

Les surfaces les plus importantes se situent dans la zone d'Entrée (environs du sondage GE1 - $11 \mathrm{~m}^{2}$ - et entrée de la Salle des Bauges - $25 \mathrm{~m}^{2}$ ). Ailleurs, elles sont moins développées : $12 \mathrm{~m}^{2}$ cumulés dans la Galerie des Mégacéros et environ $3 \mathrm{~m}^{2}$ dans le Diverticule des Ours. Dans les Galeries des Croisillons, du Cierge et du Cactus, seules des plages réduites évoquent ce faciès (tabl. 1).

Les stigmates sont le plus souvent visibles dans des zones assez étroites (Galerie des Mégacéros, Diverticule des Ours) et/ou basses (environs du sondage GE1, Galerie des Croisillons, passage de la Galerie du Cactus à celle de l'Apodémus). Un seul lieu de grandes dimensions, l'entrée de la Salle des Bauges, montre des rubéfactions à des 


\begin{tabular}{|c|c|c|c|c|c|c|}
\hline Secteur & Localisation & $\begin{array}{l}\text { Phénomènes } \\
\text { visibles }\end{array}$ & $\begin{array}{l}\text { Surfaces } \\
\text { cumulées }\end{array}$ & $\begin{array}{l}\text { Limites par } \\
\text { rapport au sol } \\
\text { actuel }\end{array}$ & $\begin{array}{l}\text { Présence et forme } \\
\text { des thermoclastes }\end{array}$ & Lien avec des charbons ou un foyer \\
\hline Secteur d'Entrée & $\begin{array}{l}\text { De la base } \\
\text { des parois au } \\
\text { plafond }\end{array}$ & $\begin{array}{l}\text { Coloration } \\
\text { rose et grise } \\
\text { Thermoclastie }\end{array}$ & $11 \mathrm{~m}^{2}$ & $\begin{array}{l}\text { Limite inférieure : } \\
0,30 \mathrm{~m} \\
\text { Limite supérieure } \\
1,45 \text { à } 2 \mathrm{~m}\end{array}$ & $\begin{array}{l}\text { Plaquettes dans les } \\
\text { couches C1 à C3 du } \\
\text { sondage }\end{array}$ & $\begin{array}{l}\text { Couche } 3 \text { base, sondage GE1 } \\
32010 \pm 390 \text { BP, } 37584 \text { à } 35283 \text { calBP (SacA14203 et } \\
\text { GifA90218 } \\
31580 \pm 370,36718 \text { à } 35123 \text { calBP (SacA14232 et } \\
\text { GifA90223) } \\
32293 \pm 380,38380 \text { à } 35661 \text { calBP (SacA11413 et } \\
\text { GifA80111) } \\
31330 \pm 270,36480 \text { à } 35130 \text { calBP (SacA11411 et } \\
\text { GifA80109) } \\
\text { Couche } 3 \text { milieu, sondage GE1 } \\
31580 \pm 280,36628 \text { à } 35224 \text { calBP (SacA11410, } \\
\text { GifA80108) }\end{array}$ \\
\hline $\begin{array}{l}\text { Entrée Salle des } \\
\text { Bauges }\end{array}$ & Plafond & $\begin{array}{l}\text { Coloration } \\
\text { rose et grise } \\
\text {. Thermoclastie }\end{array}$ & $25 \mathrm{~m}^{2}$ & $\begin{array}{l}\text { Limite supérieure } \\
\text { jusqu'à } 4 \mathrm{~m}\end{array}$ & $\begin{array}{l}\text { S'ils existent, les } \\
\text { thermoclastes sont } \\
\text { masqués par } \\
\text { l'éboulis }\end{array}$ & Aucun visible \\
\hline $\begin{array}{l}\text { Diverticule des } \\
\text { Ours }\end{array}$ & Milieu de paroi & $\begin{array}{l}\text { Coloration } \\
\text { rose et grise } \\
\text {. Thermoclastie }\end{array}$ & $3 \mathrm{~m}^{2}$ & $\begin{array}{l}\text { Limite : } 0,90 \text { à } \\
1,20 \mathrm{~m} \\
\text { Limite supérieure } \\
2 \mathrm{~m}\end{array}$ & $\begin{array}{l}\text { Rares écailles } \\
\text { minces à l'aplomb de } \\
\text { la paroi }\end{array}$ & \\
\hline Galerie des Cactus & $\begin{array}{l}\text { Voûte de } \\
\text { l'entrée de la } \\
\text { Galerie de } \\
\text { l'Apodemus }\end{array}$ & $\begin{array}{l}\text { Coloration } \\
\text { rose }\end{array}$ & $0,5 \mathrm{~m}^{2}$ & $\begin{array}{l}\text { Limite supérieure } \\
0,80 \mathrm{~cm}\end{array}$ & & \\
\hline Galerie du Cierge & Plafond & $\begin{array}{l}\text { Coloration } \\
\text { rose } \\
\text { Thermoclastie } \\
\text {. Noir de fumée }\end{array}$ & $0,5 \mathrm{~m}^{2}$ & $\begin{array}{l}\text { Limite inférieure : } \\
1,20 \mathrm{~m} \\
\text { Limite supérieure } \\
1,75 \mathrm{~m}\end{array}$ & $\begin{array}{l}\text { Quelques écailles } \\
\text { minces }\end{array}$ & $\begin{array}{l}\text { Charbon à proximité d'un petit foyer } \\
26230 \pm 280 \mathrm{BP}, 31237 \text { à } 30437 \text { (GifA 99081) } \\
\text { Valladas et al. } 2005\end{array}$ \\
\hline $\begin{array}{l}\text { Galerie du } \\
\text { Mégacéros }\end{array}$ & $\begin{array}{l}\text { Partie haute } \\
\text { des parois et } \\
\text { plafond }\end{array}$ & $\begin{array}{l}\text { Coloration } \\
\text { rose et grise } \\
\text { Thermoclastie } \\
\text { Noir de fumée }\end{array}$ & $12 \mathrm{~m}^{2}$ & $\begin{array}{l}\text { Limite supérieure } \\
1,5 \text { à } 3 \mathrm{~m}\end{array}$ & $\begin{array}{l}\text { Ecailles minces et } \\
\text { blocs } \\
\text { Certains } \\
\text { thermoclastes ont été } \\
\text { déplacés }\end{array}$ & $\begin{array}{l}\text { Charbons au sol à l'entrée de la galerie } \\
31430 \pm 420 \text { BP, } 36671 \text { à } 35030 \text { claBP (GifA99238) } \\
29740 \pm 390 \text { BP, } 35038 \text { à } 33389 \text { calBP (GifA99239) } \\
\text { Valladas et al. } 2005 \\
\text { Charbon au sol à proximité d'une sagaie } \\
32850 \pm 540,38807 \text { à } 36507 \text { calBP (GifA101459) } \\
\text { Valladas et al. } 2005\end{array}$ \\
\hline $\begin{array}{l}\text { Galerie des } \\
\text { Croisillons }\end{array}$ & Plafond & $\begin{array}{l}\text { Coloration } \\
\text { rose et grise } \\
\text { Thermoclastie }\end{array}$ & $1 \mathrm{~m}^{2}$ & $\begin{array}{l}\text { Limite supérieure } \\
1,20 \text { à } 1,40 \mathrm{~m}\end{array}$ & $\begin{array}{l}\text { Thermoclastes } \\
\text { déplacés }\end{array}$ & $\begin{array}{l}\text { Charbon en dessous du mouchage de torche GC-OO-23 } \\
31560 \pm 490,37021 \text { à } 34966 \text { (GifA101456) } \\
\text { Valladas et al. } 2005 \\
\text { Charbon au sol, sous dépôt calcité } \\
30560 \pm 370 \mathrm{BP}, 36275 \text { à } 34585 \text { calBP (GifA102566) } \\
\text { Genty et al. } 2004, \text { Valladas et al. } 2005 \\
\text { Charbons au sol sous dépôt calcité, vers le fond de la } \\
\text { galerie } \\
29180 \pm 340 \text { BP, } 34636 \text { à } 33024 \text { (GifA102568) } \\
\text { Valladas et al. } 2005\end{array}$ \\
\hline
\end{tabular}

Tableau 1 - Répartition et caractéristiques du faciès thermique. Relation avec les charbons datés.

Table 1 - Distribution and characteristics of the thermal facies. Relationship with dated charcoals.

hauteurs atteignant 3 à $4 \mathrm{~m}$. Les stigmates peuvent s'étendre sur toute l'élévation des parois, n'affecter que leur sommet et les voûtes ou seulement ces dernières (tabl. 1).

Il est bien entendu possible que d'autres endroits, non observables en raison des contraintes liées à la conservation (cheminement sur des passerelles) ou par suite de recouvrements par des concrétions récentes, aient pu être affectés, notamment dans la Salle des Bauges.

\section{Le Secteur d'Entrée et la Salle des Bauges}

Le Secteur d'Entrée se trouve dans la continuité de l'entrée paléolithique (fig. 2), obturée aux environs de $21000 \mathrm{BP}$ (Sadier et al. 2012). II se divise en deux branches, de part et d'autre d'un pilier rocheux de grandes dimensions : la branche située à l'est donne accès à la Salle Brunel tandis que l'autre, à l'ouest, débouche dans la Salle des Bauges. C'est dans cette dernière branche que les stigmates apparaissent de place en place, jusqu'au sud de la Salle des Bauges, à une distance comprise entre 15 et $40 \mathrm{~m}$ de l'entrée originelle.
Dans la galerie ouest, la rubéfaction affecte principalement le plafond, haut de 1,50 m à $2 \mathrm{~m}$, et la paroi gauche. Des plages grises sont visibles dans la zone la plus proche de la Salle des Bauges, à partir de $40 \mathrm{~cm}$ du sol (fig. 3). Les indices d'écaillage se cantonnent à la voûte, aux environs du sondage GE1 et sur la paroi gauche, à l'amont de celui-ci.

Dans la Salle des Bauges, les surfaces affectées se localisent exclusivement au plafond, jusqu'à $4 \mathrm{~m}$ de hauteur, dans de petites coupoles (diamètre inférieur au mètre). Le faciès thermique y est représenté par des changements de couleur (rose et grise) et par des plages écaillées.

\section{Le Diverticule des Ours}

II s'agit d'une rotonde de 2,50 m de diamètre et d'une hauteur maximale de $1,80 \mathrm{~m}$, qui se trouve au bout d'une galerie basse et étroite longue de $6 \mathrm{~m}$, accessible depuis la Salle Brunel à travers un chaos de blocs (fig. 2). Trois ours ont été peints sur la paroi qui fait face à l'entrée.

Seule la partie supérieure de la paroi ornée, 1,10 m à $2 \mathrm{~m}$ au-dessus du sol, présente des stigmates de chauffe. Ces 

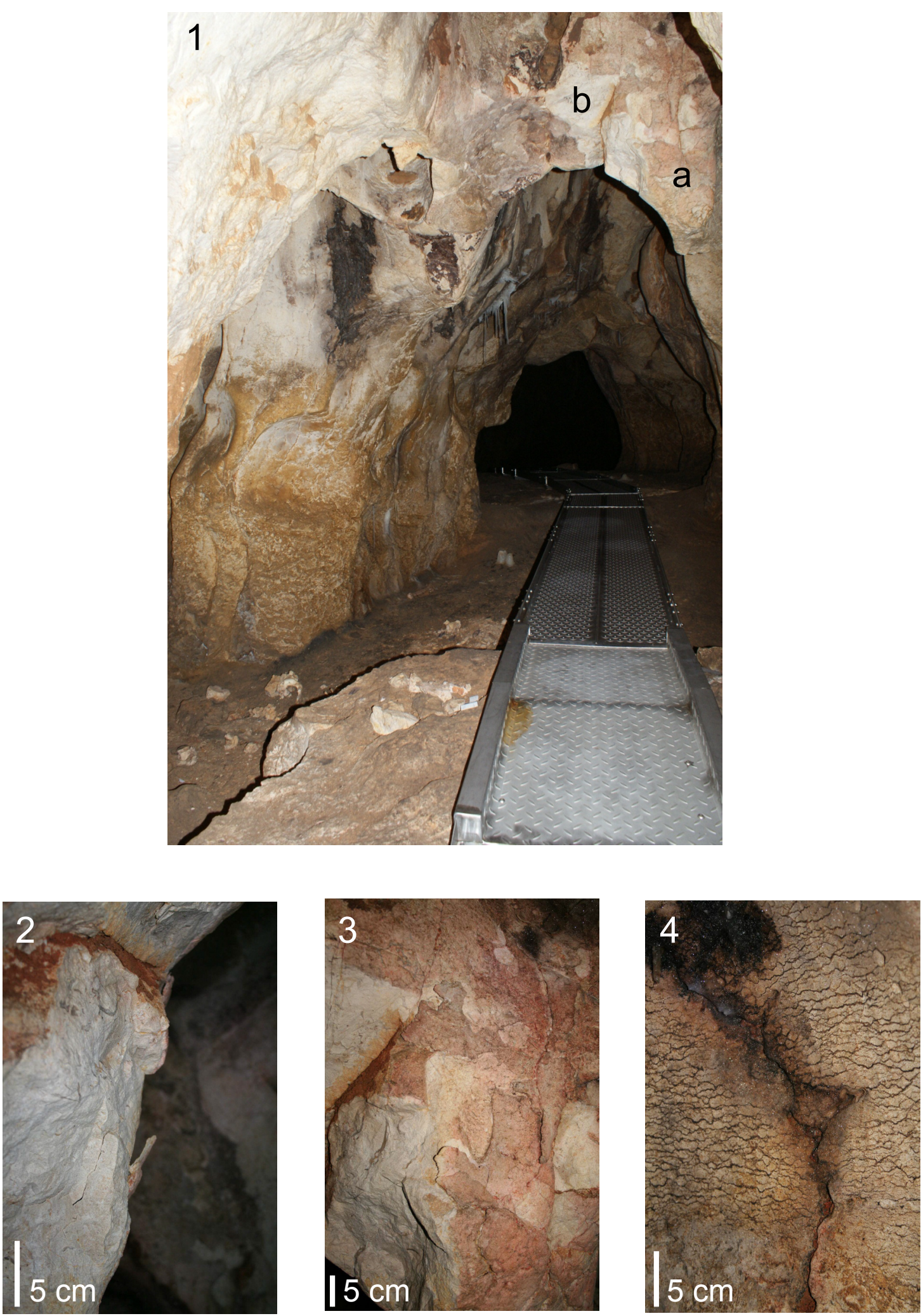

Figure 1 - Dans la galerie des Mégacéros, le faciès thermique est caractérisé par une coloration rose et grise, un écaillage superficiel de la paroi et de minces liserés noirs de carbone. 1 : faciès thermique au plafond, à l'entrée de la galerie (cliché $C$. Ferrier).

$a$ : rubéfaction, $b$ : écaillage. 2 : détail de la rubéfaction (cliché $B$. Kervazo). 3 : détail d'une des zones écaillées (cliché $B$. Kervazo). 4 : liserés noirs constitués de carbone (cliché B. Kervazo).

Figure 1- In the Galerie des Mégacéros, the thermal facies is characterized by a pink and gray colour of the rock, flakes due to the heat and thin carbon black coatings. 1: thermal facies on the vault, at the entrance of the gallery (photo C. Ferrier). a: rubefaction,

b: desquamation due to the heat. 2: detail of the rubefaction (photo B. Kervazo). 3: detail of the desquamation (photo B. Kervazo). 4: thin carbon black coatings (photo B. Kervazo). 

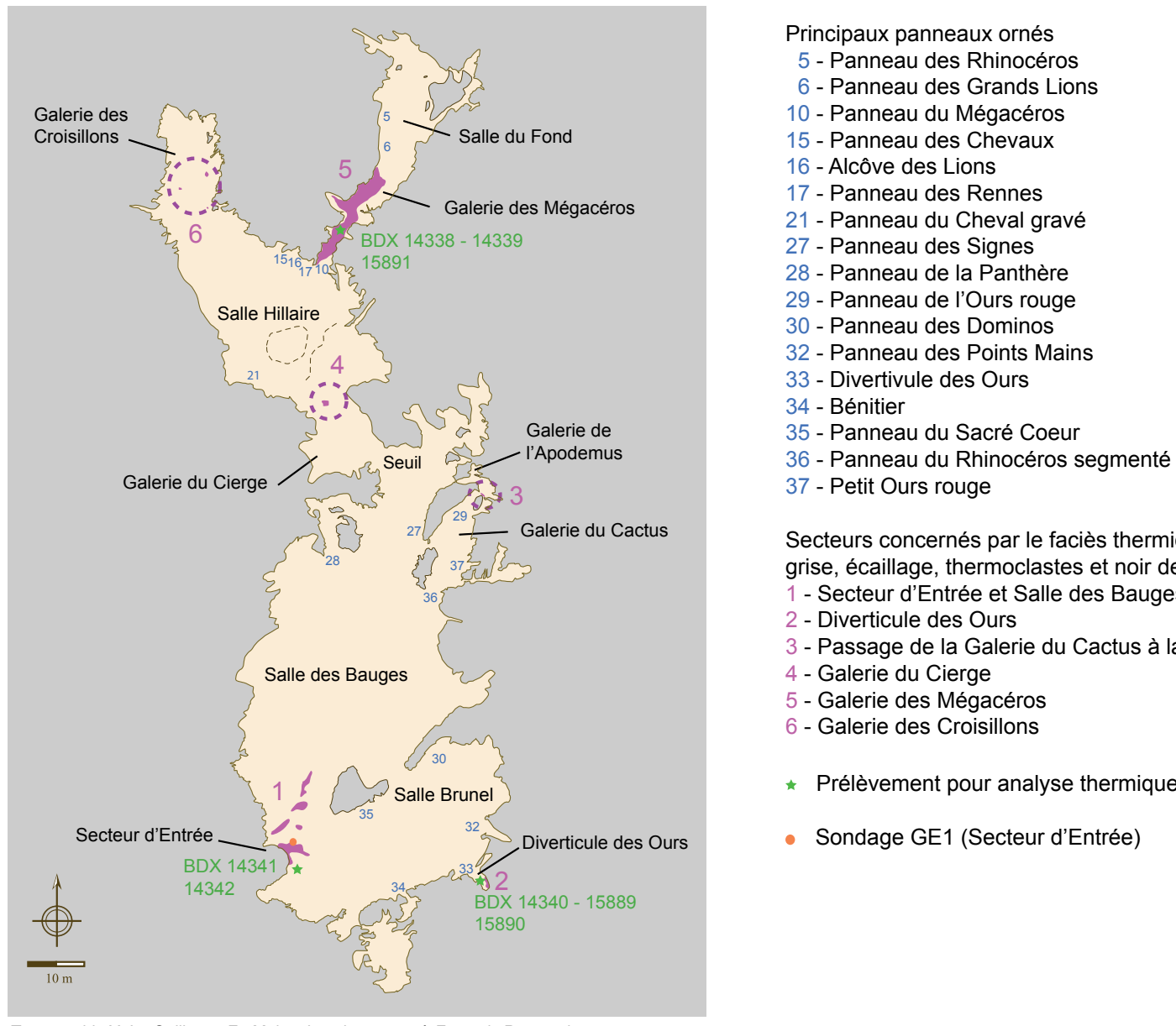

Secteurs concernés par le faciès thermique (coloration rose et grise, écaillage, thermoclastes et noir de fumée)

1 - Secteur d'Entrée et Salle des Bauges

2 - Diverticule des Ours

3 - Passage de la Galerie du Cactus à la Galerie de l'Apodemus

4 - Galerie du Cierge

5 - Galerie des Mégacéros

6 - Galerie des Croisillons

* Prélèvement pour analyse thermique

- Sondage GE1 (Secteur d'Entrée)

Topographie Y. Le Guillou et Fr. Maksud, en hommage à François Rouzaud

Figure 2 - Localisation des secteurs concernés par le faciès thermique, du sondage situé dans le secteur d'Entrée, des prélèvements pour l'étude thermique et des principaux panneaux ornés de la grotte.

Figure 2 - Location of the areas concerned with the thermal facies, of the pit located in the sector of Entrance, of the samples for the thermal study and the principal decorated panels of the cave.

derniers sont particulièrement marqués sur les deux ours situés dans la partie droite du panneau et correspondent, d'une part, à des colorations rosâtre et grise qui pénètrent de 2 à $3 \mathrm{~mm}$ dans la roche (fig. 4), d'autre part, à un écaillage guidé par les nombreuses fissures du support. Les cicatrices, parfois rubéfiées, résultent du détachement de petites écailles, épaisses de quelques millimètres, et de quelques fragments plus grands, longs d'environ $10 \mathrm{~cm}$. Le faciès thermique est également visible ponctuellement à l'entrée de la rotonde, sur la paroi gauche.

\section{Le passage de la Galerie du Cactus à la Galerie de l'Apodemus}

II correspond à un orifice, large de près de $2 \mathrm{~m}$ et haut d'environ $0,50 \mathrm{~m}$, situé au ras du sol à l'extrémité de la Galerie du Cactus (fig. 2). Deux plages rose pâle sont perceptibles juste au-dessus, sur les bords droit et gauche de la voûte. Aucun écaillage n'est visible. En raison de la distance imposée par le cheminement, il n'a pas été possible de réaliser des observations plus détaillées.

\section{La Galerie du Cierge}

Elle se localise dans la deuxième partie de la cavité, audelà du Seuil. Les stigmates de chauffe se trouvent au plafond, environ $3 \mathrm{~m}$ avant le débouché sur la Salle Hillaire, à l'aplomb de l'axe de cheminement (fig. 2). Ils affectent deux petites coupoles situées entre des pendants, à un endroit où le plafond s'abaisse jusqu'à environ 1,60 m du sol.

II s'agit de petites plages rosâtres, accompagnées par quelques très minces écailles de desquamation, parfois prêtes à tomber. Des blocs se sont détachés, à la faveur de fissures. Des revêtements noirs et des taches grisâtres de quelques millimètres de diamètre pourraient résulter de l'accumulation et de la remobilisation en vermiculations de résidus de combustion. 


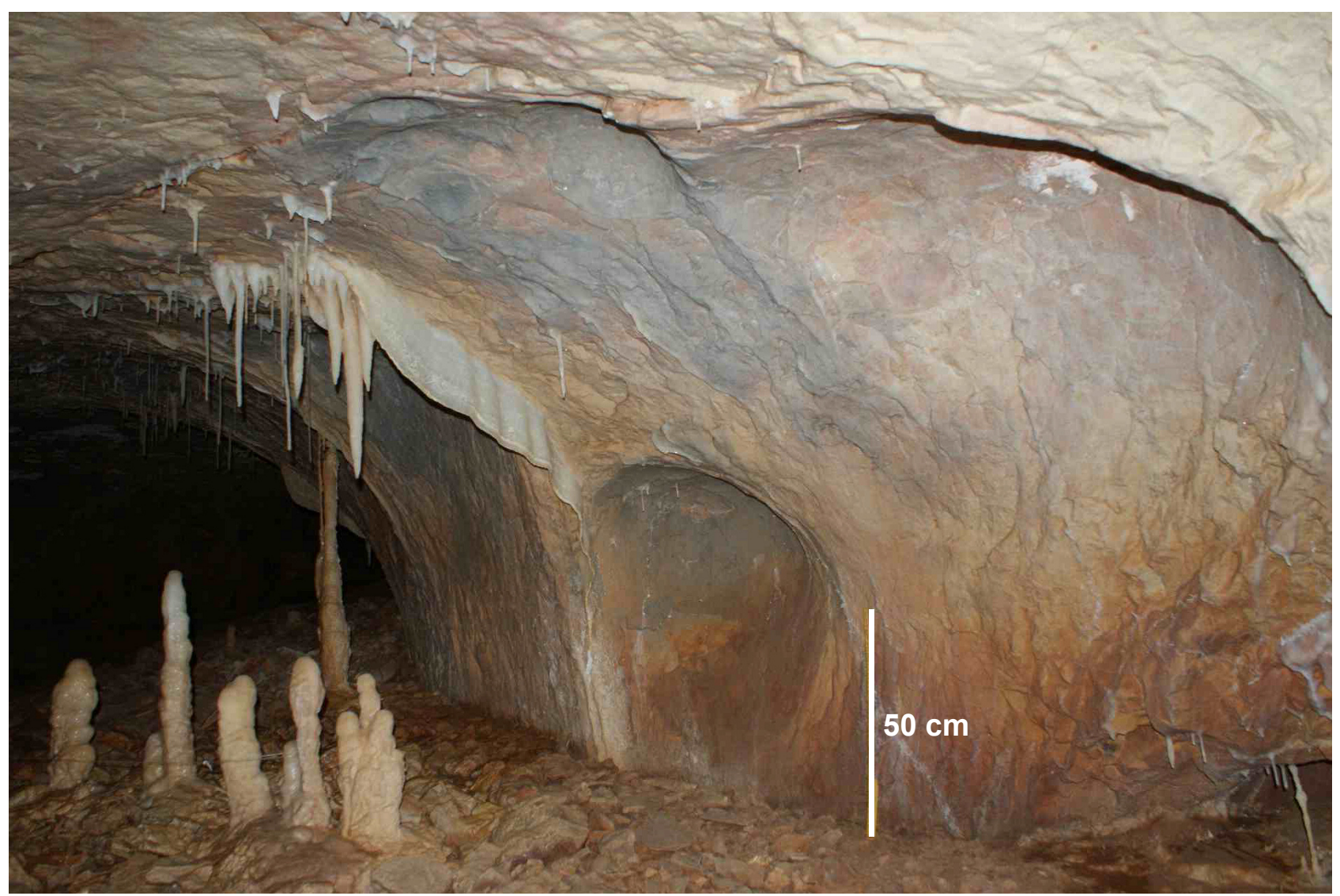

Figure 3 - Dans le Secteur d'Entrée, les traces de chauffe correspondent à des teintes rose et grise sur la paroi gauche et au plafond ainsi qu'à un écaillage, cantonné à la voûte (cliché C. Ferrier).

Figure 3 - In the Entrance area, heating traces correspond to gray and pink colours on the left wall and the ceiling. The desquamation is confined on the vault (photo $C$. Ferrier).

\section{La Galerie des Mégacéros}

II s'agit d'une galerie rectiligne de $25 \mathrm{~m}$ de longueur reliant les salles Hillaire et du Crâne à la Salle du Fond (fig. 2). Dans la première partie, la largeur est relativement faible (entre 2 et $3 \mathrm{~m}$ ) et le sol assez plat. Ensuite, à partir d'un élargissement local dû à une diaclase, la dimension du conduit atteint $6 \mathrm{~m}$ environ à sa base et le sol s'abaisse par ressauts successifs. Les parois droite et gauche portent de très abondantes marques de passage humain : dessins au charbon de bois, gravures et raclages, appuis et traces de doigts maculés par différents revêtements de paroi (argiles, pellicule d'altération...). Les griffades et les polis d'ours sont également nombreux (Feruglio et Baffier 2005 ; Philippe et Fosse 2003 ; Fosse et Philippe 2005).

Avec le Secteur d'Entrée, la Galerie des Mégacéros est l'endroit de la cavité où les marques thermiques sont les plus étendues (fig. 2). Elles se caractérisent par l'association fréquente de colorations rose et grise et par l'importance de l'écaillage. Des revêtements noirs qui pourraient correspondre à des dépôts dus à la fumée y sont également abondants (fig. 1). Cinq zones distinctes, échelonnées de l'entrée de la galerie jusqu'à son débouché dans la Salle du Fond, s'observent :

- La première, en paroi gauche, s'étend sur $3 \mathrm{~m}$ de largeur. Elle affecte la partie droite du Panneau du Rhinocéros, une cheminée qui s'élève à plus de $3 \mathrm{~m}$ et, en dessous, une petite coupole de $0,50 \mathrm{~m}$ de large située à environ $1 \mathrm{~m}$ du sol. Les teintes roses sont présentes partout, jusqu'à $3 \mathrm{~m}$ de hauteur et ceinturent des zones grises apparaissant ponctuellement près de l'entrée et dans la coupole.

La coloration pénètre d'un centimètre dans la roche à proximité des plages grises et de $0,5 \mathrm{~cm}$ ailleurs. La chauffe a également changé du brun au rouge la couleur des argiles colmatant les fissures. Cette modification pourrait être reliée à la transformation de la goethite, identifiée en $\mathrm{DRX}$, en hématite. L'écaillage couvre des surfaces importantes, surtout à l'entrée de la galerie, en association avec la coloration grise. Deux types de thermoclastes blocs et minces plaquettes - se sont détachés. Des liserés peu étendus, bruns foncés à noirâtres, ainsi qu'une tache de couleurs identiques, située à droite du panneau orné, font penser à des résidus liés à la fumée. 

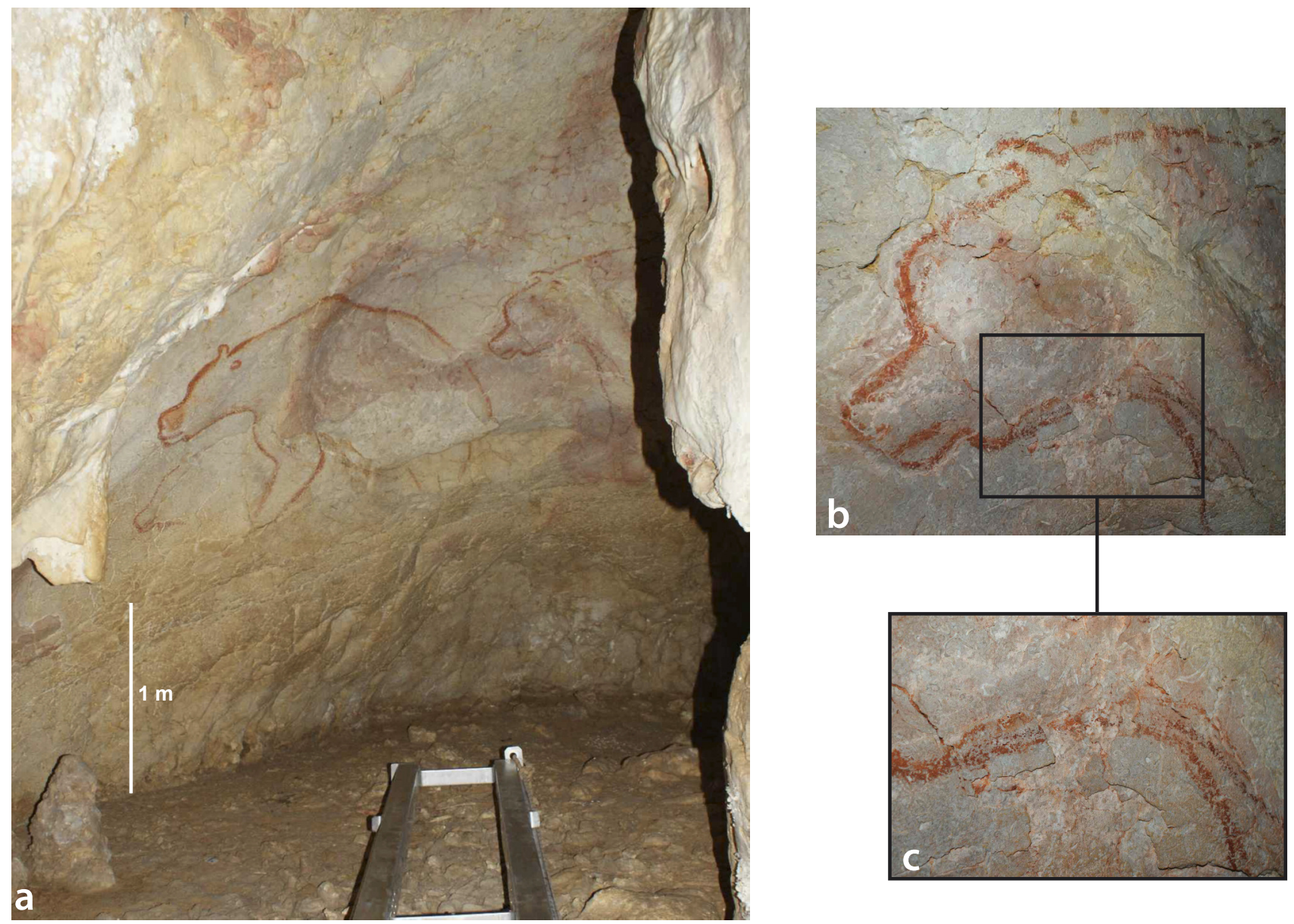

Figure 4 - Diverticule des Ours. Les ours ont été dessinés sur la paroi qui fait face à l'entrée du diverticule. a : les colorations rose et grise de la paroi, dues à la chauffe sont perceptibles dans la partie supérieure de la zone ornée. $b$ : détail de la tête de l'ours situé à droite du panneau, montrant que le dessin a été effectué après rubéfaction et écaillage de la paroi. c : le trait recouvre une cicatrice d'écaille thermique (clichés $C$. Ferrier).

Figure 4 - Diverticule des Ours. The bears were drawn on the wall which is opposite the entry. a: pink and gray colours of the wall, due to heating, are perceptible in the upper part of the decorated zone. b: detail of the head of the bear located on the right of panel, showing that the drawing was carried out after the rubefaction. c: the drawing covers a thermal scar (photos C. Ferrier).

- La deuxième zone apparaît à environ $2 \mathrm{~m}$ de la précédente, toujours en paroi gauche, au niveau d'une arête sub-verticale, creusée de grandes cupules. Elle est constituée par une plage étroite $(0,80 \mathrm{~m})$ située de $0,90 \mathrm{~m}$ à $1,60 \mathrm{~m}$ du sol. La rubéfaction et l'écaillage affectent le calcaire et, occasionnellement, en haut de paroi, un revêtement issu d'une altération phosphatée.

- Le troisième secteur se trouve juste en amont d'un ressaut déterminé par un plancher stalagmitique surplombant le sol de la seconde partie de la galerie. II s'agit du secteur le plus transformé par les feux : les stigmates s'observent au plafond et sur les deux parois, souvent dans des petites coupoles ainsi que dans des cheminées, sur 3,50 m de long et jusqu'à plus de $3 \mathrm{~m}$ de haut. La faible largeur de la galerie, de 2,50 m à $3 \mathrm{~m}$, pourrait expliquer l'intensité de l'impact thermique à cet endroit.
La rubéfaction forme des plages de dimensions variables, visibles de 1,20 m à 3,30 $\mathrm{m}$ de hauteur. La couleur grise s'observe jusqu'à la voûte. L'écaillage, sur les deux parois, a surtout produit des plaquettes minces dont certaines ne sont pas totalement dissociées. Un grand bloc (1,20 m de largeur, $2 \mathrm{~m}$ de longueur et $40 \mathrm{~cm}$ d'épaisseur), aux bords rubéfiés, s'est détaché de la paroi droite de la galerie à la faveur d'une fissure et a écrasé un squelette d'ours. Les indices de dépôts liés à la fumée correspondent à :

- des liserés horizontaux ondulés qui suivent les aspérités de la roche. Ils sont grisâtres en bas des parois, plus noirs vers le haut. Ponctuellement, ils recouvrent des zones rubéfiées ou des écailles thermiques ;

- des taches noires, sur la paroi droite, dont l'une, de $15 \mathrm{~cm}$ de diamètre, se situe dans une concavité à surface grise, recoupée par l'écaillage. 
En s'avançant vers la Salle du Fond, dans la seconde partie, plus large, de la galerie, deux zones voisines montrent des traces thermiques : la première se situe sur une avancée de la paroi gauche qui surplombe le sol d'environ $1,50 \mathrm{~m}$. La rubéfaction recouvre la partie gauche du Panneau du Félin, à l'emplacement de deux vulves, et se poursuit au plafond. Elle affecte également les argiles qui forment un mince revêtement sur la paroi et colmatent les fissures. Les dépôts rapportables à la fumée, peu marqués, en plages réduites, s'observent au-dessus du panneau orné et sur la paroi opposée. La seconde zone montre, deux mètres plus loin, deux petites taches rubéfiées, peu soutenues, de 25 et $40 \mathrm{~cm}$ de diamètre, qui se trouvent à $85 \mathrm{~cm}$ du sol, de part et d'autre du cheminement, au niveau d'un ressaut déterminé par un plancher stalagmitique. Celle en paroi droite est associée à des liserés grisâtres, analogues aux plages dont la teinte évoque des résidus de fumée dans les autres secteurs.

La dernière zone se trouve près de l'entrée de la Salle du Fond, sur un pendant de la paroi droite. La rubéfaction affecte aussi bien le calcaire qu'un revêtement d'altération phosphaté. Elle apparaît entre 1,15 m et 2,50 m de hauteur. Continue à la base, elle devient sporadique au sommet, où elle se cantonne principalement aux parties en creux. L'écaillage affecte la base du pendant et l'une de ses arêtes (fig. 5). Les thermoclastes forment des écailles d'épaisseur millimétrique à centimétrique. Les cicatrices des premières sont parfois colorées en rose. Par places, des fissures plus importantes ont déterminé la chute de fragments plus volumineux (blocs).

\section{La Galerie des Croisillons}

Dans cette galerie peu ornée, d'une dizaine de mètres de large et d'environ $25 \mathrm{~m}$ de longueur, située à l'extrémité nord de la cavité, les stigmates de feu n'ont été observés qu'au plafond (fig. 2). Les plus éloignés de l'entrée apparaissent à environ $1,40 \mathrm{~m}$ de hauteur, dans une concavité. La roche est alors écaillée, rubéfiée, avec de petites plages grises accompagnées de taches noires de plusieurs décimètres. À $5 \mathrm{~m}$ en avant et à $1,20 \mathrm{~m}$ du sol, se trouve une plage rose et grise, large d'environ $1 \mathrm{~m}$, associée à une petite écaille de desquamation. Enfin, une plage légèrement rosée s'observe à proximité de l'entrée, dans une coupole.

\section{Caractérisation par thermoluminescence de fragments de parois chauffées}

\section{Protocole d'étude}

Outre l'intensité de la coloration, l'absence de revêtement de calcite postérieur à la chauffe et la discrétion des prises d'essai ont constitué les principaux critères de prélèvement (tabl. 2). Les propriétés de luminescence des échantillons choisis ont été comparées à celle d'un calcaire de référence non chauffé, issu du même contexte géologique (tabl. 2).
Le protocole s'appuie sur celui mis en place pour la caractérisation thermique du support sédimentaire de foyers de l'âge du Bronze à la Grotte des Fraux (SaintMartin-de-Freyssengeas, Dordogne - Brodard 2013). Bien que s'adressant à des minéraux différents, la calcite à Chauvet, le quartz aux Fraux, la méthode générale demeure identique (Brodard et al. 2012) et dérive de celle, classique, de la datation par thermoluminescence (Aitken 1985). Nous reprenons en les détaillant et en les complétant les données présentées au colloque de MADAPCA de 2011 (Brodard et al. 2014). L'étape de caractérisation thermique des matériaux, fondamentale pour la datation des roches chauffées, a été particulièrement développée ici (voir entre autres Valladas 1980, 1981 ; Göksu et al. 1989 ; Koul et al. 1996 ; Roque et al. 2004a, 2004b ; Duttine et al. 2005 ; Lahaye et al. 2006 ; Guibert et al. 2009). II convient de rappeler que la calcite, bien que rarement utilisée, se prête aisément à la datation de la dernière chauffe, moyennant quelques spécificités expérimentales que nous avons mises en œuvre pour la datation de pierres calcaires chauffées à Combe Saunière et à Laugerie Haute (Roque et al. 2001a, 2001b), par rapport aux matériaux siliceux ou silicatés d'usage général.

La thermoluminescence TL repose sur les effets cumulatifs de l'irradiation par des particules et des rayonnements ionisants sur des cristaux, ici la calcite. Sous l'effet de l'irradiation ambiante, des électrons arrachés aux atomes par ionisation se retrouvent piégés par des défauts cristallins (centres-pièges). Une chauffe peut libérer tout ou partie de ces charges piégées suivant la température atteinte et sa durée. Elles peuvent alors se recombiner au niveau de centres de recombinaison (lacunes électroniques) en provoquant l'émission de photons lumineux : c'est la thermoluminescence.

Depuis les temps géologiques, des électrons se sont accumulés dans les centres-pièges induisant une saturation du remplissage. Une chauffe ancienne a pu vider partiellement ou entièrement ces pièges suivant son intensité. Par la suite, et jusqu'au moment de la mesure du signal TL par une chauffe en laboratoire, des électrons ont de nouveau été piégés sous l'effet de l'irradiation naturelle. Ce processus est à l'origine de la méthode de datation, pour laquelle l'âge de la chauffe est obtenu par le rapport de deux grandeurs physiques mesurées en laboratoire et partiellement sur le terrain, la dose archéologique, quantité d'énergie accumulée par les grains sous l'effet de la radioactivité naturelle depuis le chauffage, et la dose annuelle d'irradiation, quantité d'énergie fournie chaque année aux grains par la radioactivité naturelle supposée constante (Aitken 1985). Dans ce travail, la thermoluminescence est utilisée pour déterminer un état de chauffe.

Une première évaluation qualitative de la chauffe a été réalisée en observant l'état de saturation des signaux de la thermoluminescence naturelle de petits prélèvements de calcaire : l'ajout d'une dose importante n'accroît pas significativement la thermoluminescence d'un matériau d'origine géologique ancienne qui n'a jamais été chauffé, car l'irradiation a peuplé à saturation les centres-pièges. À 


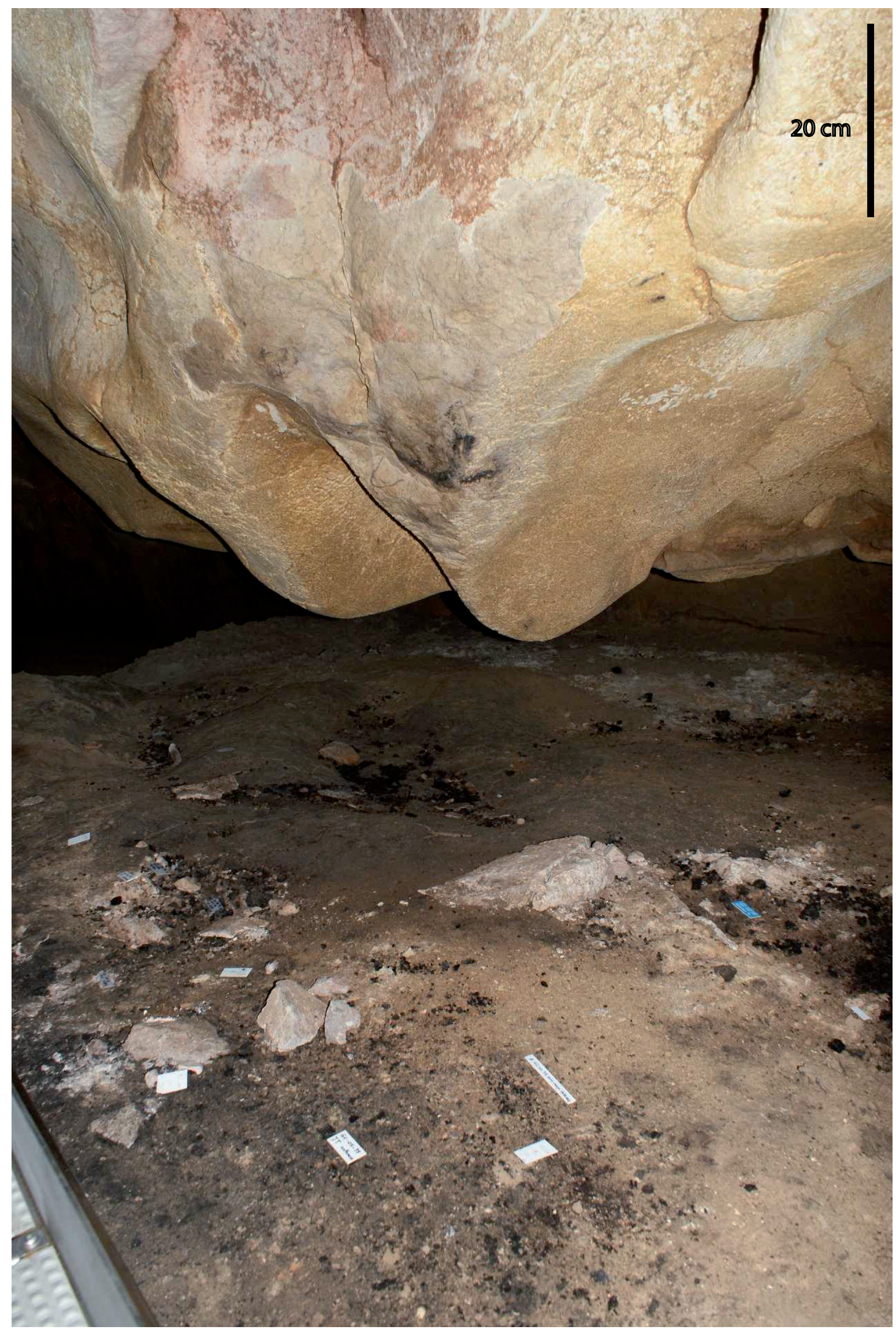

Figure 5 - Galerie des Mégacéros. Près de l'entrée de la Salle du Fond, un pendant du plafond porte des marques de rubéfaction et d'écaillage. Sur le sol, les charbons de bois sont accumulés en position secondaire dans les bauges (cliché C. Ferrier).

Figure 5 - Galerie des Mégacéros. Close to the entrance of the Salle du Fond, the ceiling shows heating marks. On the ground, the charcoals are accumulated in secondary position in the cave bear hibernation nests (photo C. Ferrier). 


\begin{tabular}{|l|c|l|}
\hline Références de l'échantillon & Localisation & \multicolumn{1}{|c|}{ Remarques } \\
\hline Galerie des Mégacéros & paroi rubéfiée & $\begin{array}{l}\text { Ecaille rubéfiée prélevée à 1,80 m au-dessus de la passerelle } \\
\text { métallique }\end{array}$ \\
\hline TL\#1-2010 / BDX 14338 & sol & $\begin{array}{l}\text { Ecaille rubéfiée prélevée sur le sol, à l'aplomb de la zone de } \\
\text { rubéfaction d'où provient l'échantillon BDX 14338 }\end{array}$ \\
\hline TL\#2-2010/ BDX 14339 & sol & $\begin{array}{l}\text { Ecaille grise prélevée au sol au pied du gros bloc sous la } \\
\text { passerelle }\end{array}$ \\
\hline TL\#3-2012/ BDX 15891 & sol & $\begin{array}{l}\text { Petite plaquette rubéfiée prélevée à proximité de l'échantillon C14 } \\
\text { GC 0803 }\end{array}$ \\
\hline Diverticule des Ours & sol & Ecaille rubéfiée prélevée au sol entre les deuX rails de circulation \\
\hline TL\#3-2010/ BDX 14340 & paroi & $\begin{array}{l}\text { Fragment du faciès rouge sur la paroi droite en entrant dans le } \\
\text { diverticule des Ours }\end{array}$ \\
\hline TL\#1-2012/ BDX 15889 & plafond & Plaquette rubéfiée extraite du plafond de l'éboulis d'entrée \\
\hline TL\#2-2012/ BDX 15890 & sol & $\begin{array}{l}\text { Plaque de calcaire non chauffée utilisée comme matériau de } \\
\text { référence pour l'étude des rubéfactions }\end{array}$ \\
\hline Eboulis d'Entrée &
\end{tabular}

Tableau 2 - Liste et localisation des échantillons prélevés pour l'étude thermique par thermoluminescence.

Table 2 - List and location of samples collected or the thermal study by thermoluminescence.



l'inverse, l'absence de saturation après irradiation au laboratoire implique que le matériau a été chauffé dans le passé. Cependant, ce test n'a de signification que si la chauffe n'est pas trop ancienne de façon à ce que la saturation ne soit pas atteinte de nouveau.

Pour les matériaux chauffés, il est possible d'aller au-delà de la seule observation présence/absence de saturation afin de déterminer une température équivalente atteinte lors du chauffage.

On a observé que pour de nombreuses espèces minérales, notamment le quartz, des états de chauffe différents produisent des variations de la forme des courbes de TL (Roque et al. 2004a, 2004b ; Brodard et al. 2012). Nous avons donc transposé notre méthodologie au calcaire de la grotte Chauvet-Pont-d'Arc. Ainsi, un fragment de calcaire urgonien non chauffé, recueilli dans la grotte, a servi de référence (BDX 14342, tabl. 2) : plusieurs échantillons ont été portés à des températures croissantes en laboratoire (de 200 à $600^{\circ} \mathrm{C}$ ) selon un protocole précis (tabl. 3). Puis leurs courbes de $T L$, régénérées par une irradiation en laboratoire (ici une dose de $68 \mathrm{~Gy}^{9}$, de l'ordre de grandeur de celle reçue depuis la chauffe supposée antérieure à $30 \mathrm{ka})$, ont été comparées à celles des matériaux archéologiques rubéfiés.

\section{Construction du référentiel thermique}

Les échantillons de référence chauffés offrent la palette de couleurs présentée en figure 6 . La rubéfaction commence dès $250^{\circ} \mathrm{C}$ et est particulièrement visible jusqu'à $375^{\circ} \mathrm{C}$, température à partir de laquelle le calcaire devient progressivement gris, à l'image des faciès observés dans plusieurs secteurs de la Grotte Chauvet-Pont d'Arc.

Les courbes TL des références thermiques obtenues (fig. 7) montrent une évolution avec la température maximale de chauffe (Tmax). II est important de signaler que les prises d'essai ont séjourné à ces températures maximales durant une heure, car les effets de la chauffe dépendent du couple durée - température du palier. Plus l'échantillon reste à une température donnée, plus les effets vont être importants car plus on laisse de temps aux processus thermodynamiquement possibles pour se produire.

Dans les prélèvements étudiés, on constate que le signal géologique est présent pour les références non chauffées ou chauffées à une température inférieure ou égale à $300^{\circ} \mathrm{C}$ (fig. 8). Au-delà de $300^{\circ} \mathrm{C}$, il n'est plus significatif et la sensibilité à l'irradiation s'affaiblit progressivement (fig. 8). On reconnaît les composantes de longue durée de

(9) Le choix de la dose administrée au laboratoire correspond approximativement à la dose naturelle acquise depuis la chauffe. La valeur donnée a été calculée en fonction de l'âge supposé et de la radioactivité des fragments de calcaire échantillonnés, mesurée par spectrométrie gamma, ici essentiellement liée à la présence d'uranium (teneur en U voisine de 4 ppm). 


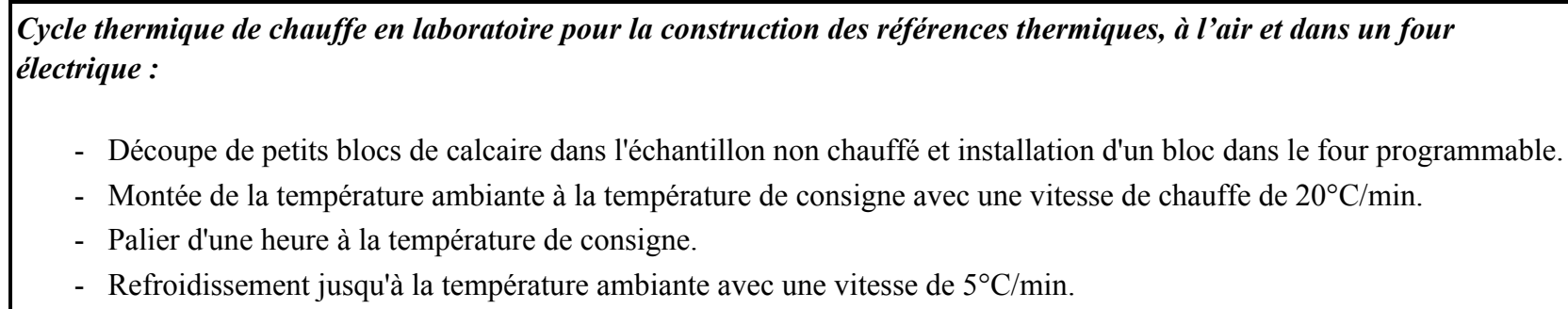

Préparation des prélèvements pour l'étude TL (échantillons archéologiques et échantillons chauffés en laboratoire en vue de la construction du référentiel thermique) :

- Broyage et tamisage : récupération de la fraction 80-200 $\mu \mathrm{m}$.

- Attaque $\mathrm{H}_{2} \mathrm{O}_{2}$ : destruction des matières organiques.

- Rinçages à l'eau déminéralisée et séchage.

- Répartition de la poudre traitée dans des coupelles en quantités aliquotes identiques d'environ 10 mg par coupelle.

\section{Conditions d'étude TL :}

- Utilisation d'un équipement de thermoluminescence développé et construit au laboratoire de l'IRAMAT-CRP2A.

- Filtrage optique MTO A520a [480 - 560 nm], filtre Infra Rouge Rejector MTO Ta2, tube photo-multiplicateur EMI 9813 QKA.

- Atmosphère de lecture sous $\mathrm{CO}_{2}$ pour éviter le phénomène de décarbonatation en surface des grains de calcite (Roque et al. , 2001a).

- Mesure du signal TL par une chauffe à $4^{\circ} \mathrm{C} / \mathrm{s}$ de la température ambiante jusqu'à $425^{\circ} \mathrm{C}$ sans palier.

Tableau 3 - Conditions expérimentales d'étude des échantillons de parois chauffées et des échantillons de référence.

Table 3 - Experimental details for the TL study of samples.

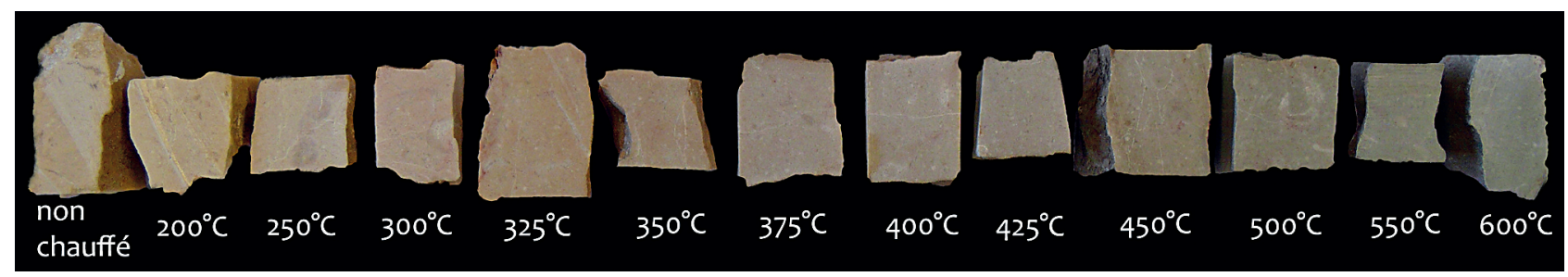

Figure 6 - Calcaire urgonien de Chauvet, éprouvettes de l'échantillon de référence BDX 14342 chauffées dans l'air pendant une heure aux températures indiquées sur la photographie (clichés A. Brodard IRAMAT-CRP2A). On notera l'évolution de la couleur.

Figure 6 - Urgonian limestone from the Chauvet Pont-d'Arc cave, cubes cut in the reference sample BDX 14342 and heated at the indicated temperatures for 1 hour in air (photo A. Brodard IRAMAT-CRP2A). Note the evolution of the colour.

vie de la $\mathrm{TL}$ de la calcite avec les pics à $280^{\circ} \mathrm{C}$ et $350^{\circ} \mathrm{C}$. À $600^{\circ} \mathrm{C}$, leur rapport d'intensité s'inverse, alors qu'il était relativement constant entre $300^{\circ} \mathrm{C}$ et $550^{\circ} \mathrm{C}$. Il est donc aisé de démontrer qu'un échantillon a été ou non chauffé. Cependant, entre $325^{\circ} \mathrm{C}$ et $550^{\circ} \mathrm{C}$, la forme des courbes n'offre pas de variation nette (le rapport d'intensité entre les émissions $\mathrm{TL}$ à $280^{\circ} \mathrm{C}$ et à $350^{\circ} \mathrm{C}$ varie peu), hormis un léger décalage de la température du maximum du second pic de $350^{\circ} \mathrm{C}$ à $360^{\circ} \mathrm{C}$ pour des chauffes supérieures à $450^{\circ} \mathrm{C}$.

\section{Étude des échantillons archéologiques rubéfiés}

L'état de saturation des signaux de thermoluminescence naturelle montre que l'accroissement de l'intensité de luminescence par ajout d'une dose de 68 Gy est net et très significatif pour les échantillons du plafond du Secteur d'Entrée et de la Galerie des Mégacéros, alors qu'il est assez faible pour celui du Diverticule des Ours, malgré un profil de la courbe de TL naturelle voisin de celui d'un échantillon chauffé à $300^{\circ} \mathrm{C}$. II semble aujourd'hui, après 


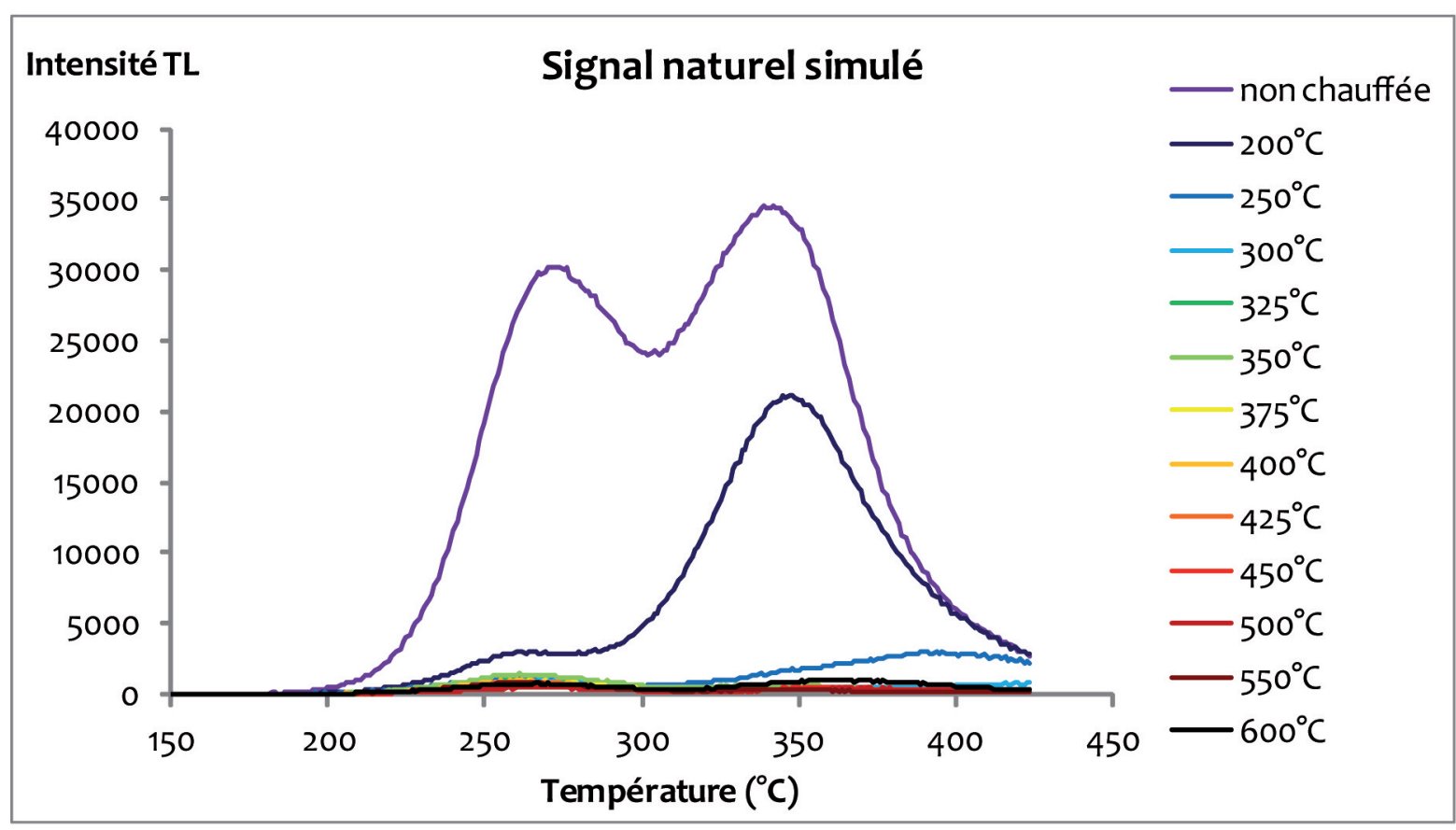

Figure 7 - Série de courbes de thermoluminescence de prises d'essai de l'échantillon géologique de référence BDX 14342 préalablement chauffées au laboratoire durant une heure dans l'air aux températures indiquées dans la légende. Les courbes de TL ont été obtenues en irradiant par une dose béta de 68 Gy les prises d'essai. Avant lecture, chaque prise d'essai subit une préchauffe consistant en un chauffage dans le four de $T L$ à $220^{\circ} \mathrm{C}$ à la vitesse de $4^{\circ} \mathrm{C} / \mathrm{s}$, suivi d'un refroidissement rapide. Cette préchauffe a pour objet de réduire les composantes de faible stabilité thermique de façon à simuler physiquement la thermoluminescence naturelle pour permettre la comparaiosn avec les échantillons archéologiquement chauffés et déterminer la paléotempérature atteinte.

Figure 7 - Set of thermoluminescence curves of aliquots of the reference sample BDX 14342 aliquots of which were submitted to a laboratory annealing process for one hour in air at the temperature given in the legend. The curves were obtained by irradiating these annealed aliquots by a beta dose (68Gy). Before the thermoluminescence measurements, every aliquot was preheated at $220^{\circ} \mathrm{C}$ following a linear ramp using a $4^{\circ} \mathrm{C} / \mathrm{s}$ heating rate, then cooled again rapidly until room temperature. This preheat process is necessary to reduce thermally unstable components in oder to simulate a natural TL signal that is compared to the natural TL signal of archaeologically heated samples to determine the attained paleotemperature. (non chauffé = unheated).

étude radiochimique (Brodard et Guibert 2012), que cet échantillon, au contact d'un sédiment assez radioactif et en présence d'une forte quantité de radon (Mangin et al. 2005) ait pu être sensiblement plus irradié naturellement qu'escompté, ce qui expliquerait à la fois le faciès "chauffé» de la TL naturelle et la faible augmentation d'intensité par l'ajout d'une dose de 68 Gy.

En comparant les courbes des échantillons rubéfiés (intensité de la luminescence et forme de la courbe) à celles des références thermiques, il est possible de préciser l'intensité de la chauffe en apportant une information sur la paléotempérature équivalente (fig. 9). L'ensemble des prélèvements étudiés présente un profil de TL naturelle similaire à celui des échantillons chauffés à plus de $300^{\circ} \mathrm{C}$, et à moins de $375^{\circ} \mathrm{C}$ si on se réfère aux couleurs. Précisons que l'atmosphère de chauffe au laboratoire est oxydante, vraisemblablement analogue en cela aux conditions de la grotte Chauvet où la roche devait être au contact de la flamme des feux paléolithiques.
Cette étude par thermoluminescence démontre bien que les parois et le plafond des zones étudiées ont subi une chauffe ancienne plutôt intense, quand on considère la masse de calcaire concernée, et que c'est cette dernière qui est responsable de la rubéfaction des calcaires.

\section{Relations avec les foyers}

Les parois portant les stigmates de chauffe ne sont généralement pas associées à des foyers visibles à la surface du sol, excepté dans les Galeries des Croisillons et des Mégacéros. Cette absence de concordance peut avoir au moins deux causes : l'érosion et le recouvrement des charbons par des dépôts, leur déplacement par les hommes et/ou les ours.

\section{Enfouissement}

Cette explication peut être invoquée principalement pour trois secteurs : le Secteur d'Entrée et la Salle des Bauges, le Diverticule des Ours, la Galerie du Cactus. 


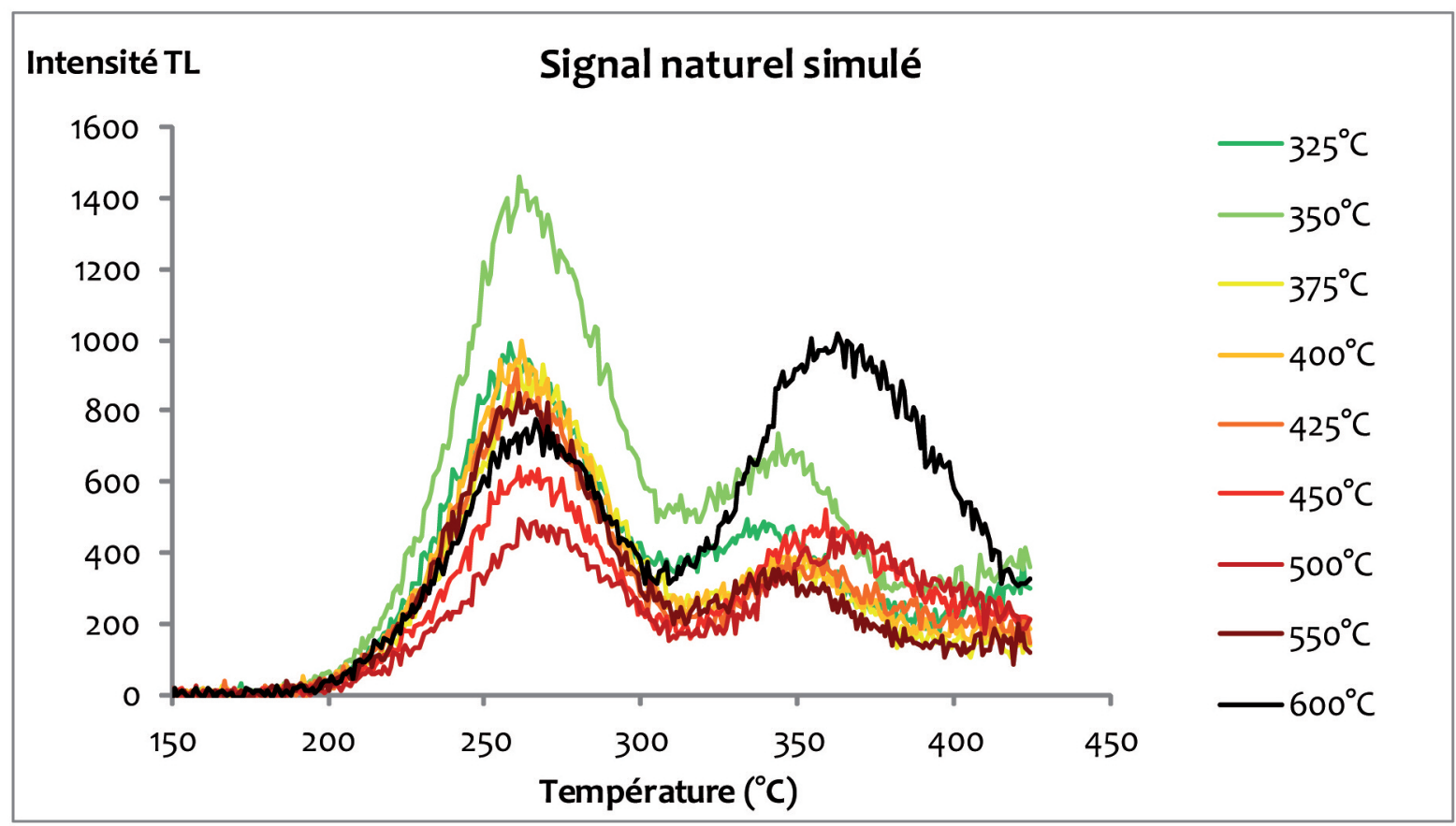

Figure 8 - Idem figure 7, détail sur les signaux de TL naturelle simulée obtenus avec les prises d'essai les plus chauffées.

Figure 8 - Same as fig. 7, zoom on simulated natural TL of high temperature annealed aliquots.

Dans le Secteur d'Entrée, un sondage réalisé dans la partie amont de l'éboulis, à proximité de la paroi portant les marques de chauffe (fig. 2), a révélé la présence, $30 \mathrm{~cm}$ sous la surface, d'un niveau riche en charbons de bois, épais de 8 à $15 \mathrm{~cm}$ (couche 3 du sondage GE1, Gély et al. 2009, fig. 10). Les charbons, contenus dans un cailloutis à matrice limono-sableuse, sont associés à des clastes portant des traces noirâtres et/ou de rubéfaction. L'étude sédimentologique a montré que ce niveau a été remobilisé par solifluxion et ruissellements (Kervazo et al. 2010). II a ensuite été recouvert par des sédiments apportés par les mêmes processus. II est donc possible que d'éventuels foyers, situés à l'aplomb des plafonds affectés par le faciès thermique, aient été remobilisés puis enfouis lors de la progression de l'éboulis vers l'entrée de la Salle des Bauges.

Dans le Diverticule des Ours, la surface de circulation paléolithique est vraisemblablement masquée par des sédiments ruisselés, pouvant provenir par exemple d'un petit conduit latéral situé en paroi gauche, à l'entrée du Diverticule. Actuellement, cette petite galerie est parcourue par des écoulements temporaires, à l'origine d'un plancher stalagmitique qui recouvre le sol.

Des traces de ruissellement sont également visibles à l'extrémité de la Galerie du Cactus. En amont, elles correspondent à des incisions peu marquées le long de la paroi droite et, en aval, à proximité de l'entrée de la Galerie de l'Apodemus, à une zone d'atterrissement. Cette organisation peut expliquer l'enfouissement d'éventuelles zones foyères.
Dans la Galerie des Mégacéros, des foyers ont pu être remaniés et les charbons enfouis par le creusement des bauges d'ours. La présence de ces derniers, entre et après les incursions humaines aurignaciennes, est attestée par les griffades et polis recoupant des dessins, sur les Panneaux du Mégacéros et des Chevaux (Feruglio et Baffier 2005 ; Fritz et Tosello 2001). Les datations C14 obtenues sur ossements, qui indiquent un arrêt de la fréquentation de la cavité par ces animaux après 33-34000 ans (cal BP), suggèrent que ces perturbations pourraient être relativement anciennes.

\section{Déplacement des charbons}

Le déplacement par l'homme de vestiges tels que des ossements d'ours ou des fragments de calcaire et de spéléothème est attesté à différents endroits de la grotte, par exemple dans les salles des Panneaux rouges et du Crâne (Geneste 2001, 2005 ; Delannoy et al. 2012). Dans la Galerie des Mégacéros, des thermoclastes ont été accumulés sous deux ressauts formés par les planchers stalagmitiques en surplomb. Leur localisation, inexplicable par un phénomène naturel, procède vraisemblablement d'un geste anthropique. Cette hypothèse peut également être avancée pour les charbons de bois de la Galerie des Mégacéros qui, lorsqu'ils sont regroupés en pied de paroi, pourraient constituer une réserve de colorant (Geneste 2005). C'est notamment le cas d'une accumulation qui se trouve sous le dessin du cheval à double crinière, en paroi gauche de la galerie. Ailleurs, la disposition des charbons indique qu'ils ont glissé le long de la pente, peut-être en raison du passage des hommes et/ou des ours, et sont restés piégés dans des creux, notamment dans des bauges (fig. 5). 


\section{Galerie des Mégacéros}

TL\#1-2012 BDX 14338
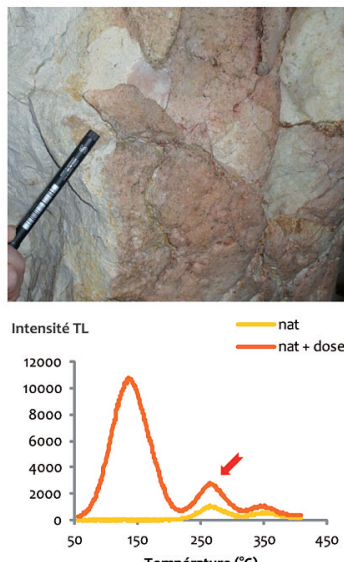

Sensibilité faible, fort accroissement du signal avec la dose ajoutée (68 Gy), coloration rouge $325^{\circ} \mathrm{C}<\mathrm{T}<400^{\circ} \mathrm{C}$

\section{Diverticule des Ours}
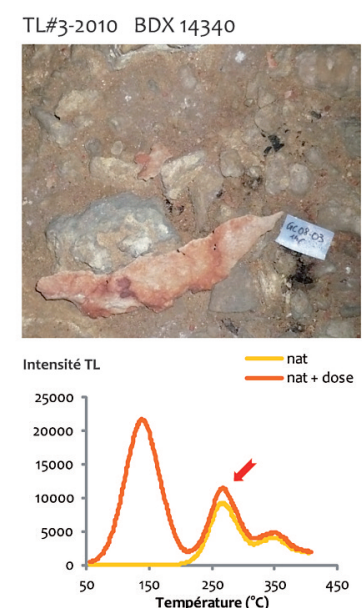

Sensibilité moyenne, faible accroissement du signal avec la dose ajoutée (68 Gy) mais forme de la courbe : échantillon chauffé; coloration rouge

$$
T \approx 300-350^{\circ} \mathrm{C}
$$

\section{Secteur de l'Entrée}
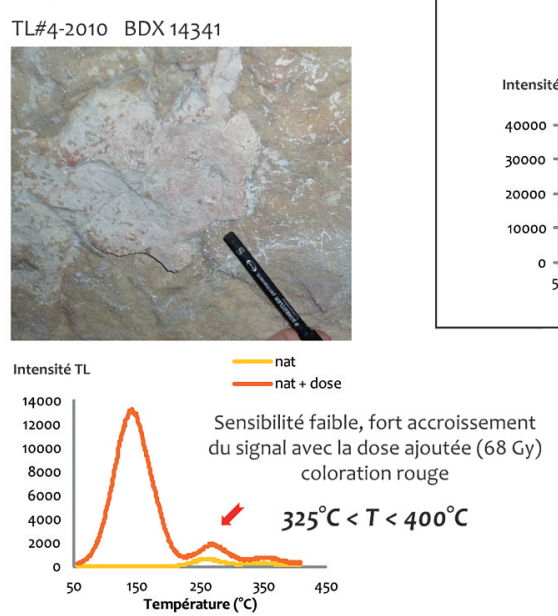

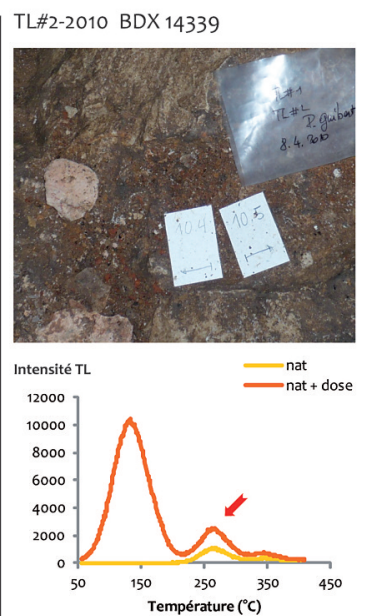

Sensibilité faible, fort accroissement du signal avec la dose ajoutée (68 Gy), coloration rouge $325^{\circ} \mathrm{C}<\mathrm{T}<400^{\circ} \mathrm{C}$

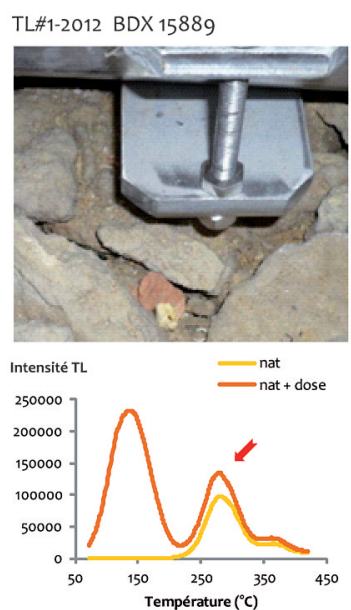

Sensibilité moyenne, faible accroissement du signal avec la dose ajoutée (100 Cy) mais forme de la courbe : échantillon chauffé ; coloration rouge

$$
T \approx 300-350^{\circ} \mathrm{C}
$$

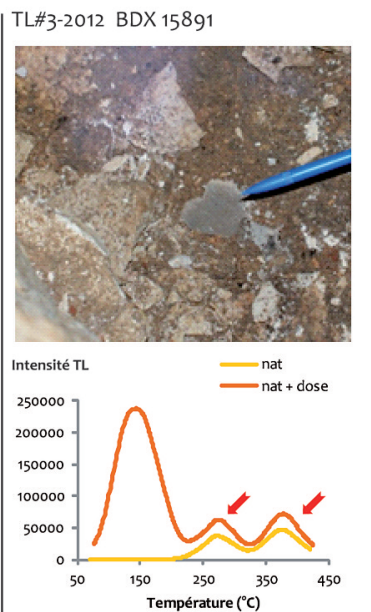

Sensibilité faible, fort accroissement du signal avec la dose ajoutée (100 Gy), intensité équivalente des 2 pics TL, décalage 2 nd pic $T L$ vers hautes $T$

$$
T>450^{\circ} \mathrm{C}
$$

TL\#2-2012 BDX 15890
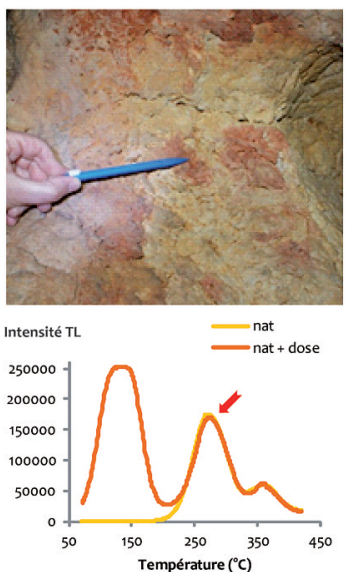

Sensibilité plus forte, pas d'accroissement du signal avec la dose ajoutée (100 Gy) mais forme de la courbe : échantillon chauffé; coloration rouge $T \approx$ ?

\section{Références}
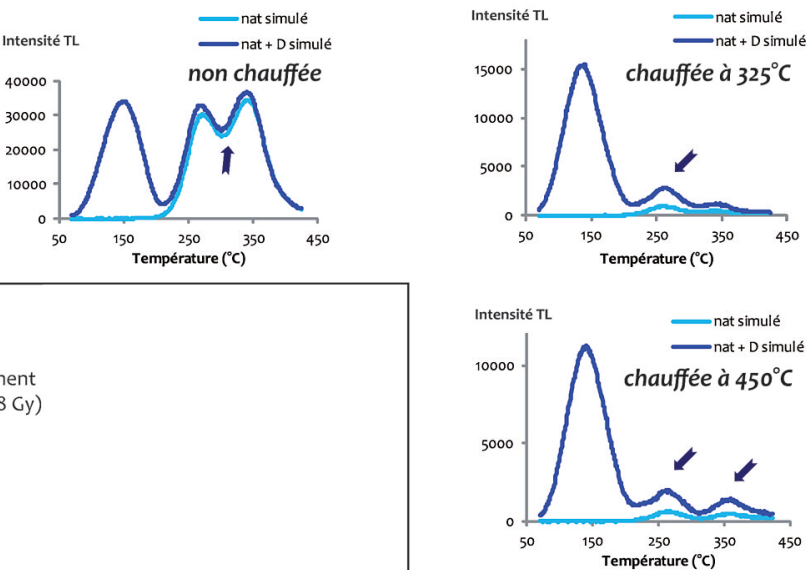

Figure 9 - Synthèse de l'étude de l'état de chauffe des échantillons rubéfiés prélevés dans la grotte Chauvet-Pont d'Arc. Figure 9 - Results of the thermal characterization of reddened samples of the Chauvet-Pont d'Arc Cave. 


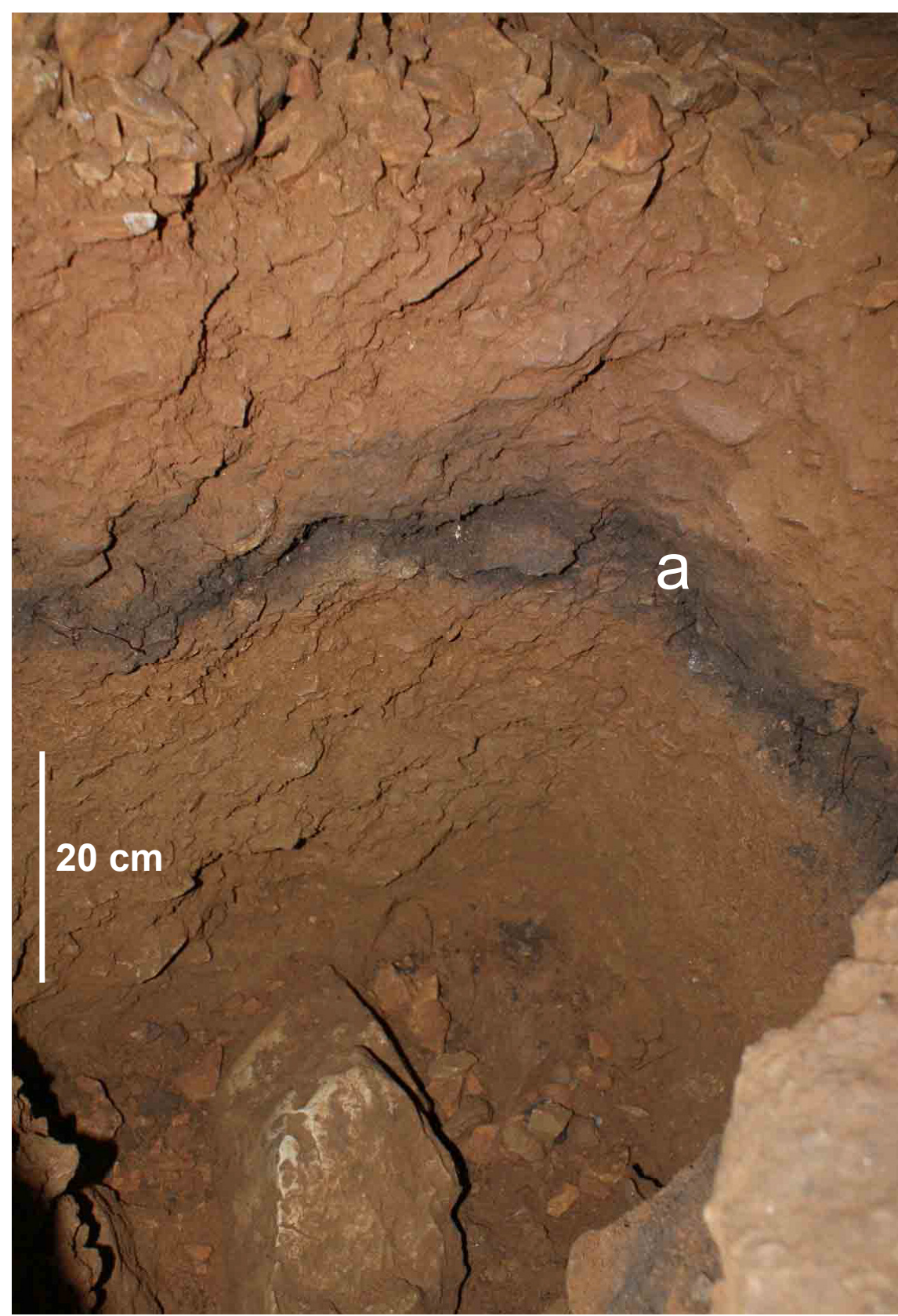

Figure 10 - Sondage GE1 effectué dans la partie amont de l'éboulis qui obstrue l'entrée de la grotte, à proximité d'une paroi portant des traces de chauffe (cliché C. Ferrier). a : niveau noir (couche 3), riche en charbons de bois, daté entre 38380 à $35661 \mathrm{cal} B P(32293 \pm 380$ $\mathrm{BP})$ et 36480 à $35130 \mathrm{cal} \mathrm{BP}(31330 \pm$ $270 \mathrm{BP})$.

Figure 10 - Pit GE1 carried out in the upper part of the a talus cone which blocks the entry of the cave, near a wall with traces of heating (photo C. Ferrier). a: the charcoal level (layer 3 ) is dated between 38380 à $35661 \mathrm{cal} B P(32293$ $+380 \mathrm{BP})$ and 36480 à $35130 \mathrm{cal} \mathrm{BP}$ $(31330 \pm 270 B P)$.

\section{Chronologie}

Les hommes ont fréquenté la grotte à l'Aurignacien et au Gravettien (Clottes 2001). Les dates obtenues sur les charbons de bois (Valladas et al. 2005 ; Cuzange et al. 2007 ; Valladas 2009) et les observations faites sur les parois ornées apportent des informations sur l'âge des feux à l'origine des stigmates thermiques, dans plusieurs secteurs.

\section{Datation C14 de concentrations charbonneuses pouvant se rapporter à des aires de combustion}

- Secteur de l'Entrée : les dates obtenues pour la couche 3 du sondage GE1 (fig. 10) sont, après calibration (Reimer et al. 2009), de $37584-35283$ cal BP (32 $010 \pm 390$ BP GifA90218) et de 36 480-35130 cal BP (31 $330 \pm 270$ BP GifA80109) (comunication privée H. Valladas). Cette partie de la grotte, où le faciès thermique affecte des surfaces importantes, est quasiment dépourvue d'œuvres pariétales (le seul élément reconnu correspond à un trait jaune).

- Galerie des Croisillons : une accumulation de charbons, dans une bauge d'ours située à l'aplomb d'une des zones rubéfiées du plafond, et interprétée comme un possible foyer (Geneste 2005), a donné une date comprise entre 36469 et 34877 cal BP $(31060 \pm 400$ BP GifA102565, Valladas et al. 2005). 


\section{Datation C14 de charbons situés à proximité des parois chauffées}

Galerie des Mégacéros : de nombreuses concentrations de charbons de bois s'observent à proximité des stigmates thermiques. Plusieurs d'entre elles ont été assimilées à des foyers (Geneste 2005), même si le sol ne montre aucune rubéfaction (Clottes 1995). Les datations C14 (Valladas et al. 2005) donnent des âges compris entre 35038-33389 cal BP $(29740 \pm 390$ BP GifA99239) et 38807-36507 cal BP $(32850 \pm 540$ BP GifA101459).

\section{Chronologie relative : relations avec les représentations pariétales}

Diverticule des Ours : la ligne de dos du grand ours, au centre du panneau, et celle du cou de l'ours situé à droite, ont été dessinées sur des cicatrices d'écailles thermiques (fig. 4), montrant que les tracés sont postérieurs à l'impact du feu. La chute des écailles s'est poursuivie plus tard, comme l'attestent l'amputation de la queue du grand ours central et la présence au sol de thermoclastes portant des ponctuations rouges. Ces ours se rapportent par leur facture aux autres ours rouges du secteur d'entrée. L'analyse génétique de la population d'ours des cavernes de la grotte Chauvet a permis de démontrer que cette espèce était en voie d'extinction, il y a 30000 ans en Ardèche et disparue au Gravettien (Bon et al. 2011). Ainsi, il serait permis de supposer que seuls des artistes aurignaciens auraient pu s'inspirer du vivant, excluant à ce titre une datation gravettienne (Elalouf et Feruglio 2012).

Galerie des Mégacéros : dans la galerie, de nombreuses marques, parfois superposées à des plages rubéfiées et à des cicatrices thermiques, n'ont pu être attribuées à l'une ou l'autre des périodes de fréquentation de la grotte : traits non figuratifs, marques de doigts et traces argileuses brunes ou orangées.

En revanche, plusieurs représentations sont rapportées à l'Aurignacien (Feruglio et al. 2005) : vulves sur le Panneau du Félin, au milieu du conduit, et rhinocéros en paroi droite, à proximité de la Salle du Fond. Elles ont été réalisées sur parois rubéfiées, c'est-à-dire après que ces dernières ont subi une chauffe.

Un mouchage de torche, qui, par analogie avec ceux datés par C14 pourrait être contemporain du Gravettien (Valladas et al. 2005), affecte la cicatrice d'une écaille thermique. Cette dernière a emporté une portion d'un signe en gerbe gravé antérieur aux dessins de l'Aurignacien qui ont été datés, à l'entrée de la galerie.

Ces différentes données chronologiques, tant absolues que relatives, semblent s'accorder pour indiquer que les feux identifiés dans le Secteur d'Entrée ainsi que dans les Galerie des Mégacéros et des Croisillons, s'intercaleraient au sein de l'Aurignacien. Cependant, il ne peut être totalement exclu que certains foyers pour lesquels on ne possède actuellement aucun repère, soient l'œuvre des Gravettiens.

\section{Conclusion}

Des parois et voûtes chauffées ont été identifiées dans différents secteurs de la grotte, depuis l'éboulis d'entrée jusqu'aux parties profondes. Excepté le Secteur d'Entrée et le début de la Salle des Bauges, il s'agit de galeries assez étroites ou basses (Diverticule des Ours, passage de la Galerie du Cactus à la Galerie de l'Apodemus, Galerie du Cierge, des Mégacéros et des Croisillons). Les espaces les plus vastes, aux plafonds très hauts, tels que les salles Brunel, des Bauges en grande partie, Hillaire et du Crâne, en sont exempts. Cependant, l'absence de trace ne permet pas d'exclure que des feux, éloignés des parois, aient pu être allumés.

Les superficies affectées par les changements de coloration et par l'écaillage, notamment dans le Secteur d'Entrée et la Galerie des Mégacéros, impliquent l'existence d'un ensemble de foyers, peut-être réalisés à des moments différents. La hauteur à laquelle se retrouvent les stigmates (jusqu'à plus de $3 \mathrm{~m}$ ) suggère qu'il s'agit de feux aux flammes particulièrement hautes. Pour le Secteur d'Entrée, le Diverticule des Ours et la Galerie des Mégacéros, les mesures par TL montrent des valeurs équivalentes à celles d'une roche ayant atteint des températures comprises entre $300^{\circ}$ et $375^{\circ} \mathrm{C}$.

Dans deux secteurs seulement, le Diverticule des Ours et la Galerie des Mégacéros, les parois modifiées par la chaleur sont directement associées à des représentations pariétales. Plusieurs indices montrent que le phénomène est antérieur à certains dessins. L'écaillage a néanmoins pu se prolonger localement, amputant une partie des œuvres (arrière-train de l'ours central dans le Diverticule des Ours, Signe en Gerbe à l'entrée de la Galerie des Mégacéros).

Dans le Secteur d'Entrée et la Galerie des Mégacéros, des charbons de bois visibles en surface ou découverts enfouis dans les sédiments peuvent être mis en relation avec les traces présentes sur les parois. Ailleurs, l'absence de ce type de vestige pourrait être par exemple expliquée par des ruissellements ou des perturbations dues à la fréquentation ursine. L'apport de combustible dans la cavité est attesté par la présence de nombreuses empreintes de branches et branchettes, prolongées parfois par une partie carbonisée, retrouvées sous le sol calcité dans la Salle Hillaire, à la surface des sédiments dans la Galerie des Mégacéros et la Salle du Fond (Garcia 2001 ; Geneste 2001). Leur abondance, qui ne peut apparemment pas s'expliquer par des circulations d'eau ou par un transport animal, montre qu'un volume important de bois a pu être apporté pour alimenter les feux dans cette partie de la cavité (Geneste 2005).

Quelles pouvaient être les fonctions des feux associés à ces stigmates ? Hormis les foyers domestiques, qui semblent pouvoir être exclus - aucune aire de combustion de ce type n'a été formellement identifiée dans la cavité -, toutes les autres propositions, déjà avancées lors de l'étude des autres grottes ornées françaises, peuvent être prises en compte : éclairage, balisage du cheminement, ravivage 
de torche, production de colorant. C'est le cas des traces ponctuelles, situées dans les Galeries du Cactus, du Cierge et des Croisillons, qui pourraient se rapporter à l'une de ces hypothèses. Deux petites plages rubéfiées, situées sur les parois, en amont d'un ressaut, dans la partie médiane de la Galerie des Mégacéros, peuvent aussi correspondre à des éclairages destinés à baliser cette difficulté du trajet. Dans la Galerie des Mégacéros, la production de colorant a été évoquée (Geneste 2001, 2005), en raison de la grande quantité de charbons dispersés sur le sol et, dans un cas, accumulés en pied de paroi sous l'une des représentations. Se pose néanmoins la question de l'utilité de feux aussi importants dans un espace aussi restreint, puisque des foyers plus modestes et dans des volumes plus grands comme par exemple la Salle Hillaire, auraient suffi pour produire les charbons nécessaires à la réalisation des panneaux noirs.

D'autres hypothèses peuvent être proposées :

- protection vis-à-vis des animaux et assainissement des sols (la grotte a été occupée par l'ours des cavernes pour hiberner pendant la période de fréquentation humaine). Ce pourrait être le cas pour les feux du Secteur d'Entrée et de la Salle des Bauges ;

- production de fumée, de chaleur ou de lumière sans nécessité pratique directe autre que celle liée au symbolique lors de la réalisation des fresques ou de la « vie » des figures par la suite ;

- recherche d'une modification volontaire de l'état de surface de la paroi, notamment de sa coloration et peut-être de sa consistance. Cette hypothèse pourrait expliquer le cas du Diverticule des Ours : les traces sont trop étendues pour un foyer d'éclairage tandis que le balisage n'a pas de sens à cette extrémité de conduit étroit. De plus, le colorant utilisé pour le dessin des ours est de l'ocre rouge et non du charbon de bois.

La grotte Chauvet-Pont d'Arc a été le siège de feux intenses qui ont teinté et desquamé ses parois et ses voûtes sur des surfaces parfois importantes. Elle constitue le premier exemple français pour lequel de telles traces ont été identifiées ${ }^{10}$. II pourrait donc être intéressant de réexaminer les grottes du Sud-Est, des Pyrénées, du Quercy et de Dordogne notamment, des stigmates ayant pu échapper aux observations, généralement focalisées sur les représentations et les sols.

\section{Remerciements}

Ce programme a bénéficié d'une aide financière spécifique du Ministère de la Culture et de la Communication. Par ailleurs, les institutions suivantes ont apporté leur soutien direct ou non, au titre des salaires, équipements, et/ou fonctionnement: CEA, CNRS, Conseil régional d'Aquitaine, Ministère de la Culture et de la Communication, Université de Bordeaux 1, Université Bordeaux 3. En outre, ce progamme a bénéficié d'un soutien du labex LaScArBx (programme Investissements d'avenir référence ANR-10LABX-52).

\section{Références bibliographiques}

AITKEN M.J. 1985 - Thermoluminescence dating. London, Academic Press, 359 p.

AMBERT P., GENDON J.-L., GALANT P., QUINIF Y., GRUNEISEN A., COLOMER A., DAINAT D., BEAUMES B., REQUIRAND C. 2005 - Attribution des gravures paléolithiques de la grotte d'Aldène (Cesseras, Hérault) à l'Aurignacien par la datation des remplissages géologiques. C. R. Palevol, 4, p. 275-284.

AZÉMA M., GÉLY B., BOURRILLON R, GALANT P. 2012 - L'art paléolithique de Baume Latrone (France, Gard) : nouveaux éléments de datations. Inora, 64, p. 6-12.

BAFFIER D., GIRARD M. 1998 - Les cavernes d'Arcy-surCure. Paris, La maison des roches, $119 \mathrm{p}$.

BEGOUËN R., CLOTTES J., GIRAUD J.-P., ROUZAUD F. 1989 - Les foyers de la caverne d'Enlène (MontesquieuAventès, Ariège). In : M. Olive, Y. Taborin dir., Actes du colloque International de Nemours « Nature et fonction des foyers préhistoriques ", 12-13-14 mai 1987, Mémoires du Musée de Préhistoire d'Île de France, éd. A.P.R.A.I.F, Nemours, 2, p. 165-179.

BEGOUËN R., FRITZ C., TOSELLO G., CLOTTES J., PASTOORS A., FAIST F., BOURGES F., FOSSE P., LACOMBE S., LANGLAIS M. 2009 - Le sanctuaire secret des bisons. II y a 14000 ans, dans la caverne du Tuc d'Audoubert. Paris, Somogy édition d'art, $415 \mathrm{p}$.

BODU P., JULIEN M., VALENTIN B., DEBOUT G. et al. 2006 - Un dernier hiver à Pincevent : les Magdaléniens du niveau IV0. Gallia Préhistoire, 48, p. 1-180.

BON C., BERTHONAUD V., FOSSE P., GÉLY B., MAKSUD F., VITALIS R., PHILIPPE M., VAN DER PLICHT J., ELALOUF J.-M. 2011 - Low regional diversity of late cave bears mitochondrial DNA at the time of Chauvet Aurignacian paintings. Journal of Archaeological Science, 38, p. 1886-1895.

(10) Pour apporter des informations complémentaires aidant à comprendre la signification de ces traces de chauffe, un programme de feux expérimentaux en domaine souterrain a été réalisé dans une carrière de Gironde (IThEM : Les traces de feux dans la grotte Chauvet : Des Impacts Thermiques sur les parois à la caractérisation des feux. Expérimentation et Modélisation). II a permis de comprendre le fonctionnement d'un foyer dans une cavité, d'appréhender son impact sur la paroi, de mesurer les températures associées (air et roche) et d'étudier le comportement des fumées. Ce programme a été porté conjointement par les UMR 5060IRAMAT-CRP2A et 5199-PACEA. II a bénéficié, via le LaScArBx, d'une aide de l'État gérée par l'Agence Nationale de la Recherche au titre du programme Investissements d'avenir portant la référence ANR-10-LABX-52. 
BRODARD A., GUIBERT P., LÉVÊQUE F., MATHÉ V., CAROZZA L., BURENS A. 2012 - Thermal characterization of ancient hearths from the cave of Les Fraux (Dordogne, France) by thermoluminescence and magnetic susceptibility measurements. Quaternary Geochronology, 10, p. 353-358, (doi:10.1016/j.quageo.2012.04.013).

BRODARD A. 2013 - Caractérisation thermique de structures de combustion par les effets de la chauffe sur les minéraux : thermoluminescence et propriétés magnétiques de foyers de la grotte des Fraux. Thèse de Doctorat de l'Université Michel de Montaigne Bordeaux 3.

BRODARD A., GUIBERT P., FERRIER C., DEBARD E., KERVAZO B., GENESTE J.-M. 2014 - Les rubéfactions des parois de la grotte Chauvet : une histoire de chauffe ? Actes du colloque MADAPCA, Micro Analyses et Datations de l'Art Préhistorique dans son Contexte Archéologique, MNHN-C2RMF, 16-18 novembre 2011, Paleo, special issue, 2014, p. 233-235.

CHAUVET J.-M., BRUNEL-DESCHAMP É., HILLAIRE C. 1995 - La grotte Chauvet à Vallon-Pont-d'Arc. Paris, Éditions du Seuil, coll. Arts Rupestres, 118 p.

CLOTTES J. 1977 - Les découvertes pariétales de ces dernières années dans les cavernes de Niaux et de Fontanet (Ariège), Bulletin de la Société préhistorique française, t. $74, \mathrm{n}^{\circ} 1$, p. 6.

CLOTTES J. 1995 - Postface. La grotte Chauvet aujourd'hui. In : Chauvet J.-M., Brunel-Deschamp É., Hillaire C., La grotte Chauvet à Vallon-Pont-d'Arc. Paris, Éditions du Seuil, coll. Arts Rupestres, p. 81-118.

CLOTTES J. dir. - 2001 - La grotte Chauvet. L'art des origines. Paris, Éditions du Seuil, coll. Arts Rupestres, 224 p.

CLOTTES J., COURTIN J., VANRELL L. 2005 - Cosquer redécouvert. Paris, Éditions du Seuil, 255 p.

COMBIER J. 1984 - Grotte de la Tête du Lion. In : L'art des cavernes. Atlas des grottes ornées paléolithiques françaises, Imprimerie nationale, p. 595-599.

COMBIER 1989 - À propos de la chronologie de l'art pariétal rhodamien. In : L'Art pariétal paléolihique. Périgueux-Le Thot, novembre 1984, Actes des colloques de la Direction du Patrimoine, p. 115-116.

CUZANGE M.-T., DELQUE-KOLIC, GOSLART T., GROOTES P.M., HIGHAM T.F.G., KALTNECKER E., NADEAU M.-J., OBERLIN C., PATERNE M., VAN DER PLICHT J., BRONK RAMSEY C., VALLADAS $H$., CLOTTES J., GENESTE J.-M. 2007 - Radiocarbon Intercomparison Program for Chauvet Cave. Radiocarbon, vol. 49, 2, p. 339-347.

DEBARD E., FERRIER C., KERVAZO B. 2012 - Étude géologique de la grotte Chauvet-Pont d'Arc. Bilan des travaux de la triennale 2010-2012. In : "Études pluridisciplinaires à la grotte Chauvet-Pont d'Arc (Ardèche). Rapport d'activité 2010-2012 », vol. 1, p. 59-98.
DELANNOY J.-J., GENESTE J.-M., DAVID B., KATHERINE M., GUNN R. G., WHEAR R. L. 2012 - Apport de la géomorphologie dans l'aménagement et la construction sociale des sites préhistoriques. Exemples de la grotte Chauvet-Pont d'Arc (France) et de Nawarla Gabarnmang (Australie), Paleo, 23, p. 85-104.

DELIBRIAS G., EVIN J. 1980 - Sommaire des datations ${ }^{14} \mathrm{C}$ concernant la Préhistoire en France. Dates parues de 1974 à 1978. Bull. Soc. préhist. française, t. 77, 7, p. 215-224.

DUTTINE M., GUIBERT P., PERRAUT A., LAHAYE C., BECHTEL F., VILLENEUVE G. 2005 - Effects of thermal treatments on TL and EPR of flints and their importance in TL-dating: application to french Mousterian sites of Les Forêts (Dordogne) and Jiboui (Drôme). Radiation Measurements, 39/4, p. 375-385.

ELALOUF J.-M., FERUGLIO V. 2012 - L'ours spéléologue. Pour la Science, $n^{\circ} 412$, p. 28-33.

FERRIER C., DEBARD E., KERVAZO B., AUJOULAT N., BAFFIER D., FERUGLIO V., FRITZ C., GÉLY B., GENESTE J.-M., KONIK S., LACANETTE D., LASTENNET R., MAKSUD F., MALAURENT P., PLASSARD F., TOSELLO G. 2012 - Approche taphonomique des parois des grottes ornées. In : J. Clottes. (dir.), L'art pléistocène dans le monde / Pleistocene art of the world / Arte pleistoceno en el mundo, Actes du Congrès IFRAO, Tarascon-sur-Ariège, septembre 2010, Symposium " Datation et taphonomie de l'art pléistocène ", Préhistoire, Arts et Sociétés, Bulletin de la Société Préhistorique Ariège-Pyrénées, LXV-LXVI, 20102011, Livre synthèses : p. 192, CD : p. 1071.

FERUGLIO V., BAFFIER D. 2005 - Les dessins noirs des salles Hillaire et du Crâne, une chronologie relative. In : J.M. Geneste (dir.), La grotte Chauvet à Vallon-Pont-d'Arc : un bilan des recherches pluridisciplinaires. Actes de la séance de la Société préhistorique française, Lyon, 2003. Paris : Édition Société préhistorique française (Travaux 6) Fédération française de spéléologie et Association française de karstologie (Karstologia, Mémoire 11), p. 149158, [Paru simultanément et sous le même titre dans Bulletin de la Société préhistorique française, 102, 1, p. 149-158.]

FOSSE P., PHILIPPE M. 2005 - La faune de la grotte Chauvet : paléobiologie et anthropozoologie. In : J.-M. Geneste (dir.), La grotte Chauvet à Vallon-Pont-d'Arc : un bilan des recherches pluridisciplinaires. Actes de la séance de la Société préhistorique française, Lyon, 2003. Paris : Édition Société préhistorique française (Travaux 6) Fédération française de spéléologie et Association française de karstologie (Karstologia, Mémoire 11), p. 89102, [Paru simultanément et sous le même titre dans Bulletin de la Société préhistorique française, 102, 1, p. 149-158.]

FOUCHER P., WATTEZ J., GEBHARDT A., MUSCH J. 2000 - Les structures de combustion mésolithiques de la Pierre-Saint-Louis (Geay, Charente-Maritime). Paleo, 12, p. 165-200. 
FRITZ C., TOSELLO G. 2001 - Le secteur des chevaux. In : J. Clottes dir., La grotte Chauvet. L'art des origines. Paris, Éditions du Seuil, coll. Arts Rupestres, p. 106-117.

GARCIA M.-A. 2001 - Les empreintes et les traces humaines et animales. In : J. Clottes dir., La grotte Chauvet. L'art des origines. Paris, Éditions du Seuil, coll. Arts Rupestres, p. 34-43.

GÉLY B. 2000 - Grottes ornées de l'Ardèche. L'art des cavernes. Éditions le Dauphiné libéré, 52 p.

GÉLY B., MAKSUD F. 2009 - Sondage GE-1 dans la galerie d'entrée - Travaux 2009 et synthèse des résultats 2006-2009. In : « Etudes pluridisciplinaires à la grotte Chauvet-Pont d'Arc (Ardèche). Rapport d'activité triennal 2007-2009 et perspectives 2010-2012 », p. 211-233.

GENESTE J.-M. 2001 - La fréquentation et les activités humaines. In : J. Clottes dir., La grotte Chauvet, l'art des Origines, Paris, Éditions du Seuil, coll. Arts rupestres, p. 44-50.

GENESTE J.-M. 2005 - L'archéologie des vestiges matériels dans la grotte Chauvet : paléobiologie et anthropozoologie. In : J.-M. Geneste dir., La grotte Chauvet à Vallon-Pont d'Arc : un bilan des recherches pluridisciplinaires, Actes de la séance de la Société préhistorique française, Lyon 2003, Paris : Édition Société préhistorique française (Travaux 6) Fédération française de spéléologie et Association française de karstologie (Karstologia, Mémoire 11), p. 135-144, [Paru simultanément et sous le même titre dans Bulletin de la Société préhistorique française, 102, 1, p. 149-158.]

GÖKSU, H.Y., WEISER, A., REGULLA D.F. $1989-110^{\circ} \mathrm{C}$ TL peak records the ancient heat treatment of flint. Ancient $T L, 7$ (1), p. 15-17.

GUIBERT P., BAILIFF I. K., BLAIN S., GUELI A.M., MARTINI M., SIBILIA E., STELLA G., TROJA S.O. 2009 Luminescence dating of architectural ceramics from an early medieval abbey: the St-Philbert intercomparison (LoireAtlantique, France). Radiation Measurements, 44, 5-6, p. 488-493.

GUILBERT R. 2001 - "Le Sansonnet" et "Les Agnels" (Vaucluse), un exemple de fragmentation thermique intentionnelle du silex au Sauveterrien. Paleo, 13, p. 245-250.

KERVAZO B., DEBARD E., FERRIER C., CHAMPAGNON B., PANCZER G., RAFFAELLY-VESLIN L. 2010 - Étude géologique de la grotte Chauvet-Pont d'Arc. Rapport 2010. In : Études pluridisciplinaires à la grotte Chauvet-Pont d'Arc (Ardèche). Rapport d'activité 2010, p. 115-164.

KOUL, D.K., SINGHVI, A.K., NAMBI, K.S.V., BHAT, C.L., GUPTA P.K. 1996 - Feasibility of estimating firing temperature using the $110^{\circ} \mathrm{C}$ TL peak of quartz. Applied Radiation and Isotopes, 47 (2), p. 191-194.
LAHAYE C., GODFREY-SMITH D. I., GUIBERT P., BECHTEL F. 2006 - Equivalent thermal history of ferrigunous sandstones based on the thermal activation characteristics of quartz. Radiation Measurements, 41, p. 995-1000.

OMNÈS J. 1982 - Labastide. Grotte ornée paléolithique des Hautes-Pyrénées. Lourdes, J. Omnès, imprimerie Lendrat, 352 p.

MANDEVILLE M. D. 1973 - A consideration of the thermal pretreatment of chert. Plains Anthropologist, t. 1, n. 61, p. $177-200$.

MANGIN A., D'HULST D., BOURGES F. 2005 - Étude de l'environnement souterrain de la grotte Chauvet, suivi annuel des concentrations en radon. Rapport auprès de la Conservation Régionale des Monuments Historiques de Rhône-Alpes, 11 p.

PHILIPPE M., FOSSE P. 2003. — La Faune de la Grotte Chauvet (Vallon-Pont-d'Arc, Ardèche) : présentation préliminaire paléontologique et taphonomique. Paleo, 15, p. $123-140$

PHILIPPE M., MONNEY J., ARGANT J., CHAUVET J.-M., DEBARD E., GÉLY B., LATEUR N., STOCCHETTI S. 2009 - La grotte ornée paléolithique des Deux-Ouvertures (Saint-Martin-d'Ardèche). Ardèche archéologie, 26, p 3-12.

REIMER P.J., BAILLIE M.G.L, BARD E, BAYLISS A, BECK, J.W. BLACKWELL P.G., BRONK RAMSEY C., BUCK C.E., BURR G.S., EDWARDS R.L., FRIEDRICH M., GROOTES P.M., GUILDERSON T.P., HAJDAS I., HEATON T.J., HOGG A.G., HUGHEN K.A., KAISER K.F., KROMER B., MCCORMAC F.G., MANNING S.W., REIMER R.W., RICHARDS D.A., SOUTHON J.R., TALAMO S., TURNEY C.S., VAN DER PLICHT J., WEYHENMEYER C.E. 2009 - IntCal09 and Marine09 radiocarbon age calibration curves, $0-50,000$ years cal BP, Radiocarbon, 51, 4, p. 1111-1150.

ROQUE C., GUIBERT P., VARTANIAN E., BECHTEL F., SCHVOERER M. 2001a - Thermoluminescence - dating of calcite: study of heated limestone fragments from Upper Paleolithic layers at Combe-Saunière, Dordogne, France. Quaternary Geochronology (Quaternary Science Review), 20, p. $935-938$.

ROQUE C., GUIBERT P., VARTANIAN E., BECHTEL F., OBERLIN C., EVIN J., MERCIER N., VALLADAS H., TEXIER J.-P., RIGAUD J.-PH., DELPECH F., CLEYETMERLE J.-J., TURQ A. 2001b - Une expérience de croisement de datations $\mathrm{TL} / 14 \mathrm{C}$ pour la séquence solutréenne de Laugerie-Haute, Dordogne. In : Datation, $X X l^{e}$ Rencontres Internationales d'Archéologie et d'Histoire d'Antibes, Dir. J.-N. Barrandon, P. Guibert, et V. Michel, Editions APDCA, Antibes, 217-232. 
ROQUE C., GUIBERT P., VARTANIAN E., VIEILLEVIGNE E., BECHTEL F. 2004a - Changes in luminescence properties induced by thermal treatments; a case study at Sipan and Trujillo Moche sites (Peru). Radiation Measurements, 38-1, p. 119-126.

ROQUE C., GUIBERT P., DUTTINE M., VARTANIAN E., CHAPOULIE R., BECHTEL F. 2004b - Dependence of luminescence characteristics of irradiated quartz with thermal treatment and consequences for TL-dating: Application to Dating of Solutrean heated quartz pebbles from Laugerie Haute (Dordogne, France). Geochronometria 23, p. 1-8.

SADIER B., DELANNOY J.-J., BENEDETTI L., BOURLÈS L., JAILLET S., GENESTE J.-M., LEBATARD A.-E., ARNOLD M. 2012 - Further constraints on the Chauvet cave artwork elaboration. PNAS, USA, 109, p. 8002-8006.

VALLADAS H. 1980 - Investigation of thermoluminescence of burnt sandstones from prehistoric sites. Nuclear Instruments and Methods, 175, p. 230-232.

VALLADAS H. 1981 - Thermoluminescence de grès de foyers préhistoriques : estimation de leur température de chauffe. Archaeometry, 23, p. 221-229.
VALLADAS H., TISNERAT-LABORDE N., CACHIER H., KALTNECKER E., ARNOLD M., OBERLIN C., EVIN J. 2005 - Bilan des datations carbone 14 effectuées sur des charbons de bois de la grotte Chauvet. In : J.-M. Geneste (dir.), La grotte Chauvet à Vallon-Pont-d'Arc : un bilan des recherches pluridisciplinaires. Actes de la séance de la Société préhistorique française, Lyon, 2003. Paris : Édition Société préhistorique française (Travaux 6) - Fédération française de spéléologie et Association française de karstologie (Karstologia, Mémoire 11), p. 109-113, [Paru simultanément et sous le même titre dans Bulletin de la Société préhistorique française, 102, 1, p. 109-113.]

VALLADAS H. 2009 - Bilan de l'étude chronologique des occupations préhistoriques de la grotte Chauvet. Bilan 2009 (LSCE). In : « Études pluridisciplinaires à la grotte ChauvetPont d'Arc (Ardèche). Rapports d'activités triennaux 20072009 et Perspectives 2010-2012 », p. 437-442.

WAHL L. 1973 - Découverte d'un nouveau réseau préhistorique dans la grotte de Fontanet à Ornolac-Ussatles-Bains (Ariège). Spelunca, $4^{\mathrm{e}}$ série, p. 91-92.

WEISGERBER G., WILLIES L. 2001 - The Use of Fire in Prehistoric and Acient Mining: Firesetting. Paleorient, vol. 26/2, p. 131-149. 